\title{
Direct numerical simulations in solid mechanics for understanding the macroscale effects of microscale material variability
}

\author{
Joseph E. Bishop ${ }^{\mathrm{a}, *}$, John M. Emery ${ }^{\mathrm{a}}$, Richard V. Field ${ }^{\mathrm{a}}$, Christopher R. Weinberger ${ }^{\mathrm{b}}$, David J. \\ Littlewood $^{\mathrm{c}}$ \\ ${ }^{a}$ Engineering Sciences Center, Sandia National Laboratories, Albuquerque, NM 87185, U.S.A. \\ ${ }^{b}$ Department of Mechanical Engineering and Mechanics, Drexel University, Philadelphia, PA 19104, U.S.A. \\ ${ }^{c}$ Computing Research Center, Sandia National Laboratories, Albuquerque, NM 87185, U.S.A.
}

\begin{abstract}
A fundamental challenge for the quantification of uncertainty in solid mechanics is understanding how microscale material variability is manifested at the macroscale. In an era of petascale computing and future exascale computing, it is now possible to perform direct numerical simulations (DNS) in solid mechanics where the microstructure is modeled directly in a macroscale structure. Using this DNS capability, we investigate the macroscale response of polycrystalline microstructures and the accuracy of homogenization theory for upscaling the microscale response. Using a massively parallel finite-element code, we perform an ensemble of direct numerical simulations in which polycrystalline microstructures are embedded throughout a macroscale structure. The largest simulations model approximately 420 thousand grains within an I-beam. The inherently random DNS results are compared with corresponding simulations based on the deterministic governing equations and material properties obtained from homogenization theory. Evidence is sought for both surface effects and other higher-order effects as predicted by homogenization theory for macroscale structures containing finite microstructures.
\end{abstract}

Keywords: material variability, direct numerical simulation, polycrystal, homogenization, multiscale, Voronoi, uncertainty quantification, stainless steel 304L

\footnotetext{
*corresponding author

Email addresses: jebisho@sandia.gov (Joseph E. Bishop), jmemery@sandia.gov (John M. Emery), rvfield@sandia.gov (Richard V. Field), cweinberger@coe.drexel.edu (Christopher R. Weinberger), djlittl@sandia.gov (David J. Littlewood)
} 


\section{Introduction}

Engineered structures composed of metallic materials typically contain complex spatially varying polycrystalline microstructures resulting from the solidification process as well as a series of manufacturing processes such as casting, metal forming (e.g., stamping, forging, rolling), and fabrication (e.g., welding, machining). These manufacturing processes not only alter the initial microstructure but also create complex spatially varying texture (nonuniformly-random crystal orientations) [1,2]. A key challenge in quantifying uncertainty in solid mechanics is understanding how the process-dependent microscale material variability is manifested at the macroscale in engineering quantities of interest.

In an era of petascale computing and future exascale computing [3], it is now possible to perform direct numerical simulations (DNS) in solid mechanics, in analogy to DNS turbulence modeling in fluid mechanics, where the microstructure is modeled directly in a macroscale structure. Using this DNS capability, we investigate the macroscale response of polycrystalline microstructures and the accuracy of homogenization theory for upscaling the microscale response. We perform an ensemble of 100 direct numerical simulations in which polycrystalline microstructures are embedded throughout a macroscale structure. The microstructural embedding is accomplished through the use of a voxelation approach and a highly refined finite-element mesh of the macroscale structure with element sizes several times smaller than the grain size. Each finite element is assigned the properties of the grain containing the centroid of the hexahedral element. This approach to microstructural embedding is simple and robust, unlike an explicit microstructural meshing approach in which degenerate elements are invariably created making it difficult to simulate many realizations of the microstructure $[4,5,6,7,8]$. In this work, we are less interested in detailed stresses near the grain boundaries, but rather with the stress fluctuations above the grain scale.

Depending upon the relative size of the microstructure (e.g., grain size) and the macroscale structural feature (e.g., fillet radius, hole radius, section thickness), the local stress and strain fields may violate the assumption of scale separation, a key assumption in homogenization theory [9]. As noted by Mindlin [10]:

"Higher-order effects can be expected to come into play in linear-elastic solids when the representative length scale of the deformation field becomes comparable to a micro-structural length scale." 
Homogenization theory, at least for periodic media, predicts the existence of higher-order gradient effects in both the governing field equations and constitutive relations whenever the microstructure is finite $[9,11,12,13]$. For an infinitesimally small microstructure (first-order homogenization theory), these higher-order effects vanish. Also, homogenization theory predicts the existence of a surface effect, or boundary layer, due to the difference in material confinement at the surface as compared to the interior $[14,15,16]$. This boundary layer arises due to the break in the periodicity assumption at the surface and due to the satisfaction of displacement or traction boundary conditions using the homogenized material properties. For an infinitesimally small microstructure, the thickness of this boundary layer vanishes. Additionally, Beran and McCoy [17] and Drugan and Willis [18] have shown that the governing field equations for the ensemble-averaged stress field are nonlocal with the extent of nonlocality governed by the microstructural correlation length. When the correlation length is infinitesimally small, the governing field equations for the ensemble-averaged stress field become local in character, and the ensemble-averaged stress field equals the stress field resulting from the first-order homogenized field equations. Using the ensemble of DNS results, we search for evidence of these effects.

The example macroscale structure is an I-beam with holes in the web region and fillets connecting the web and flange. The I-beam is quasi-statically loaded in a torsional mode to create stress gradients throughout the structure along with active stress concentrations in the web region. The example material is stainless steel 304L which possesses an austenitic (FCC) microstructure. For this material, each grain possesses a relatively large elastic anisotropy ratio, making it a seemingly ideal material to display higher-order effects. The microstructure is idealized as a Voronoi tessellation seeded through a maximal Poisson disk sampling process. This seeding process is also referred to as random close-packing [19]. The seeding process and subsequent Voronoi tessellation results in an equiaxed grain structure. The crystal orientation of each grain is assumed to be uniformly random (no texture) and uncorrelated with neighboring grains. This microstructure is in stark contrast to a composite material containing a periodic microstructure with perfect correlation between periodic cells. For the periodic case, higher-order homogenization effects are known to exist $[11,12,13]$.

In order to explore the effect of a finite grain size and the assumption of scale separation in the homogenized solution, two ratios of web-thickness to grain size are studied: four and eight. For the case of four grains through the web-thickness, each realization of the I-beam contains 
approximately 60 thousand grains. For the case of eight grains through the web-thickness, each realization of the I-beam contains approximately 420 thousand grains. While only the ratio of grain size to structural size is important for the linear elastic case studied here, we note that for grain sizes on the order of $100 \mu \mathrm{m}$, there are approximately 1000 grains per cubic millimeter and 1 million grains per cubic centimeter. Thus, the number of grains considered in this work is still relatively small for structures consisting of many cubic centimeters of material or relatively small grains sizes. Expected future advances in computer resources will only increase the volume of material analyzable by direct numerical simulation [3]. This will be discussed in more detail in Section 3 .

Recently, Temizer and Wriggers [20] have explored the embedding of periodic microstructures within macroscale 2D and 3D structures at local regions experiencing large strain gradients, such as contact points and crack tips, in order to quantify the error resulting from the assumption of scale separation. Our goals here are similar, however, here we embed a random polycrystalline microstructure throughout the entire macroscale structure.

This paper is organized as follows. Homogenization theory is briefly reviewed in Section 2. The Direct Numerical Simulation modeling approach is presented in Section 3. In Section 4 the idealized microstructure is defined and the effective homogeneous material properties are obtained. The direct numerical simulation results of an I-beam are presented in Section 5. Conclusions are given in Section 6.

\section{Overview of homogenization theory}

Homogenization is the mathematical process of replacing a heterogeneous material with a fictitious homogeneous material whose macroscopic response is equal to that of the true heterogeneous material. The material properties of the fictitious homogeneous material are called the effective properties. The homogenized material is the result of a limiting process in which the microstructural length scale approaches zero. In practice, the microstructure is finite, but as long as there is a "scale separation" between the macroscale response and the microstructural length scale, the response of the homogenized material is a good approximation to the true heterogeneous material.

Equality between the true heterogeneous material and the fictitious homogeneous material is defined using the mathematical concepts of strong and weak convergence [9]. A sequence of 
functions $\left(u_{n}\right), n=1,2,3, \ldots$, with $u_{n} \in L^{2}$, converges strongly to $u \in L^{2}$ if

$$
\lim _{n \rightarrow \infty}\left\|u_{n}-u\right\|=0
$$

and converges weakly if

$$
\lim _{n \rightarrow \infty}\left\langle u_{n}, v\right\rangle=\langle u, v\rangle \quad \forall v \in L^{2} .
$$

Here $L^{2}$ represents the space of square-integrable functions, $\langle u, v\rangle$ represents the $L^{2}$ inner product, and $\|u\|=\sqrt{\langle u, u\rangle}$ is the norm induced by the inner product. For an example, consider the sequence of functions

$$
u_{n}(x)=\bar{u}(x)+\hat{u}_{n}(x) \quad x \in[0,1], \quad n=1,2,3, \ldots,
$$

where

$$
\bar{u}(x)=4 x(1-x) \quad \text { and } \quad \hat{u}_{n}(x)=\frac{1}{n} \sin (n \pi x) .
$$

It can be shown that $u_{n}(x)$ converges strongly to $\bar{u}(x)$, but $u_{n}^{\prime}(x)$ converges only weakly to $\bar{u}^{\prime}(x)$, where ( $)^{\prime}$ denotes differentiation with respect to $x$. The functions $u_{n}(x), \bar{u}(x)$ and their derivatives are shown in Figure 1 for several values of $n$. Here, $n \rightarrow \infty$ is analogous to $\epsilon \rightarrow 0$ where $\epsilon$ represents the maximum grain size; $u_{n}(x)$ represents the displacement field in a hypothetical heterogeneous material, and $\bar{u}(x)$ is the displacement field of the homogenized material.

The mathematical homogenization of boundary value problems consisting of materials with periodic microstructure has been well studied [21, 22, 9, 23]. One of the main results is that the displacement field of the macroscopic boundary value problem containing the heterogeneous material converges strongly to the displacement field of the macroscopic boundary value problem containing the homogenized material. Furthermore, the strain (stress) field of the macroscopic boundary value problem containing the heterogeneous material converges weakly to the strain (stress) field of the macroscopic boundary value problem containing the homogenized material. These results have been extended to random microstructures by Papanicolaou and Varadhan [24] using probabilistic definitions of convergence.

In the limit of an infinitesimally small microstructure, the effective material properties of the homogenized material are well defined, i.e., independent of the boundary conditions, for both periodic and random microstructures $[22,23]$. It is important to note that for random microstructures, the effective material properties are deterministic. This will be discussed in more detail in Section 4.2 along with the determination of effective material properties for the model 

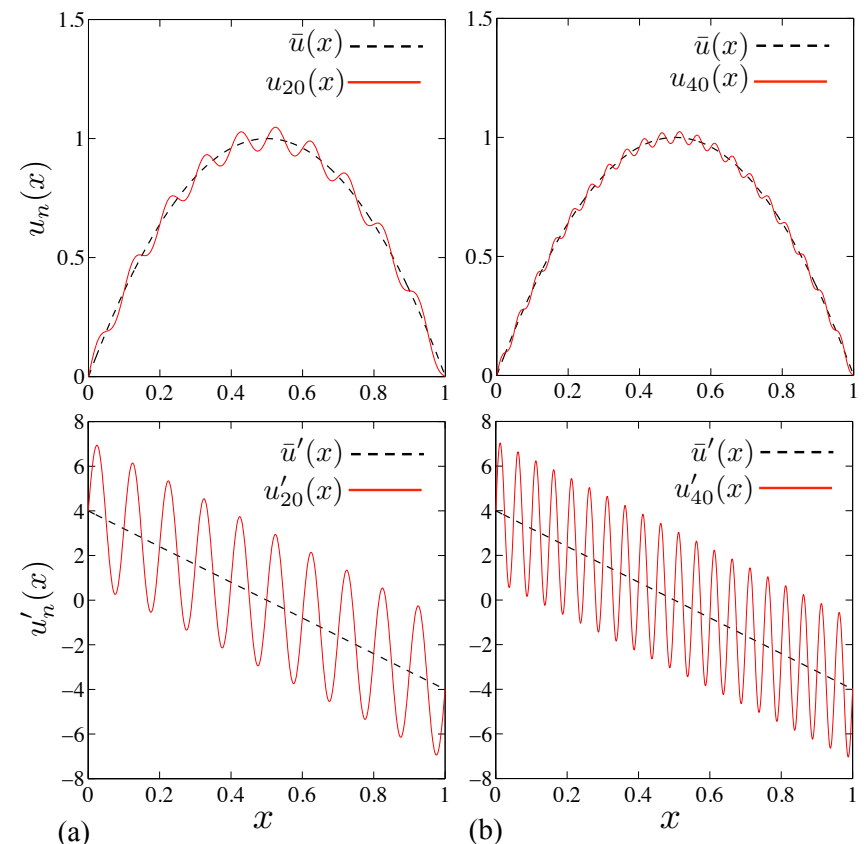

(b)

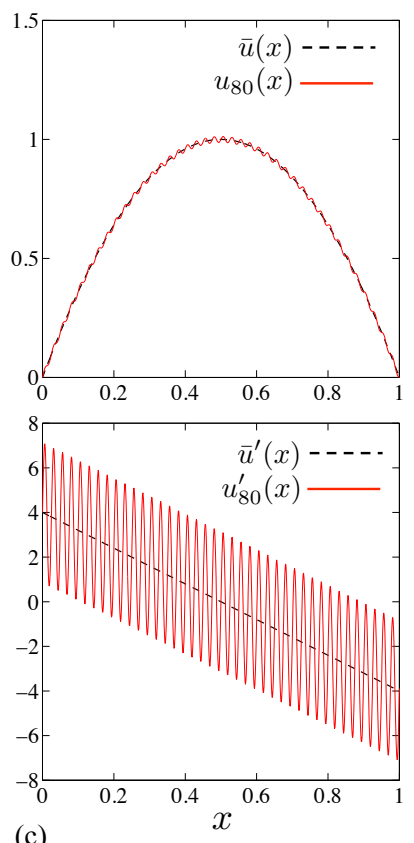

(c)

Figure 1: Example of both strong (top row) and weak convergence (bottom row). The function $u_{n}(x)=\bar{u}(x)+\hat{u}_{n}(x)$ converges strongly to the function $\bar{u}(x)$ as $n \rightarrow \infty$. However, the derivative of $u_{n}(x)$ converges only weakly to the derivative of $\bar{u}(x)$. Here, $\bar{u}(x)=4 x(1-x)$ and $\hat{u}_{n}(x)=\frac{1}{n} \sin (n \pi x)$. (a) $n=20$, (b) $n=40$, (c) $n=80$. 
microstructure defined in Section 4.1. For a periodic microstructure, additional strain gradient terms arise in the balance of linear momentum whenever the microstructural size is finite with respect to the macroscale length scale $[12,25,13]$. Additional higher-order material properties will exist as well. It is unclear whether these formulations can be extended to structures with a random, but finite, microstructure. However, a field theory of the ensemble-average behavior of structures with random microstructure has been obtained by Beran and McCoy [17] and Drugan and Willis [18]. This will be discussed in Section 5.5.

\section{Direct numerical simulations}

The explicit meshing of polycrystalline grains of general morphology, using a mesh that conforms to the grain boundaries, is tedious and far from robust $[4,5,6,7,8]$. Such meshes typically require regularization of the grain geometry and are mainly obtained using tetrahedral finite elements. These tetrahedral meshes typically contain degenerate elements (an element with an edge of nearly zero length or a collapsed element with a relatively small volume), and are not robust in the large deformation regime. Even with the use of tetrahedral meshes, a given microstructural realization typically requires user intervention, and the likelihood of requiring user intervention increases as the number of grains increases. One goal of this work is to perform direct numerical simulations on a large number of microstructural realizations (an ensemble). Each realization contains hundreds of thousands of grains. Thus, a robust discretization approach is needed. For this reason, we adopt an approach in which the macroscale structure is meshed explicitly with hexahedral finite elements, but the microstructure is only pixelated/voxelated (using the same mesh). This is a common technique in the modeling of composite and polycrystalline microstructures within a representative volume element [26, 27]. Pyle, et al., have compared the use of a voxelation representation of the grain structure to the use of a conformal tetrahedral mesh in polycrystal simulations [28]. One of their conclusions was that the texture evolution and statistical distribution of stress was very similar for both representations, in addition to the macroscopic behavior. In this work, we are less interested in detailed stresses near the grain boundaries, but rather with the stress fluctuations above the grain scale. With this goal in mind, a voxel representation of the grain structure is deemed sufficient.

To model the microstructure directly within a macroscale structure, we leverage high performance computing resources and use a finite-element mesh that is fine enough to resolve the mi- 

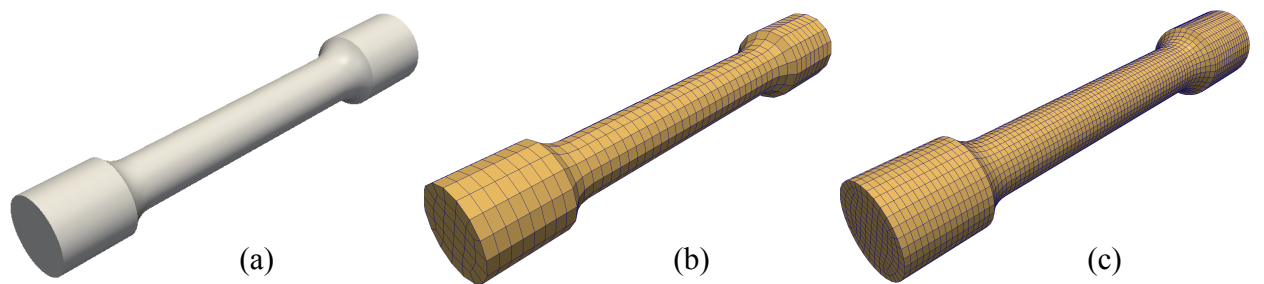

Figure 2: (a) Example structure, a round-bar tension specimen. (b) Base hexahedral mesh denoted as $r 0$. (c) Subsequent hexahedral meshes, $r 1, r 2, r 3, \ldots$ are obtained from uniform hierarchical refinement in which each hexahedron is divided into 8 . New surface nodes created during each refinement are located on the exact geometry. Here, $r 1$ is shown.

crostructural detail. A volume of $1 \mathrm{~cm}^{3}$ containing $100 \mu \mathrm{m}$ sized grains contains approximately 1 million grains. The direct numerical simulation of the microstructure within this volume requires many tens of millions of finite elements to resolve the grain structure. The use of this highly refined mesh requires massively parallel finite-element software and for an implicit solution, an equation solver that can scale to thousands of processors. Here we use the implicit solid mechanics module within the Sierra multiphysics finite-element software suite [29] and the FETI-DP solver. FETI-DP is a domain-decomposition iterative solver that uses Lagrange multipliers to enforce compatibility at the subdomain interfaces [30].

For an example of the DNS modeling approach, consider the example structure shown in Figure 2(a), a round-bar tension specimen. First, a coarse conformal hexahedral mesh of the macroscale structure is created as shown in Figure 2(b). This base mesh is referred to as the $r 0$-mesh refinement. Subsequent hexahedral mesh refinements, $r 1, r 2, r 3, \ldots$ are obtained from uniform hierarchical refinement in which each hexahedron is divided into 8 hexahedra. New surface nodes created during each refinement are located on the exact geometry. Figure 2(c) shows the $r$ 1-mesh refinement. The hierarchical refinement is continued until the element size is sufficiently small to resolve each grain to the desired level of accuracy. In this work, the meshing tool CUBIT [31] used to create both the geometry and hexahedral meshes.

Next, a bounding box of the macroscale structure is created. The model microstructure is then created within this bounding box. This is demonstrated in Figure 3 using only the gauge section of the round-bar tension specimen. The model microstructure is the idealized equiaxed Voronoi microstructure developed in Section 4.1. Each finite element is then assigned to a specific grain based on the location of the centroid of the hexahedral element. The resulting grain imprinting is shown in Figure 4 for several levels of mesh refinement. For this example, there 


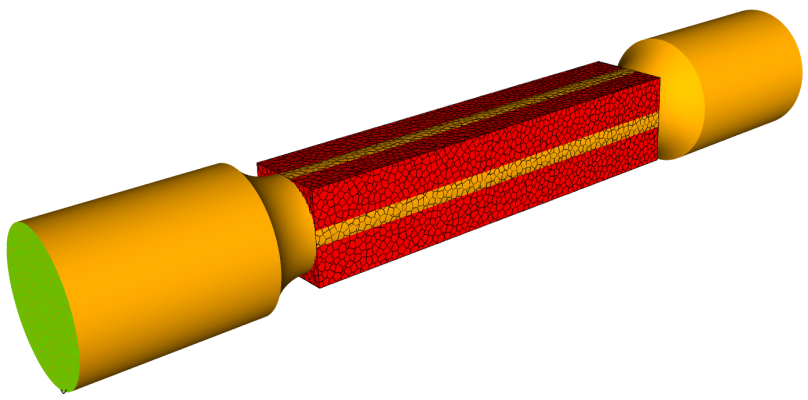

Figure 3: Imprinting of a microstructure onto a macroscopic structure, here the gauge section of the round-bar tension specimen shown in Figure 2. The Voronoi microstructure shown in Figure 6 is used for the imprinting. This imprinting is done implicitly by identifying the grain that contains a given hexahedral centroid. The results are shown in Figure 4.

are approximately six grains through the diameter of the gauge section. The base $r 0$ hexahedral mesh barely resolves the grain structure with one element per grain, approximately. Mesh refinements $r 1$ through $r 4$ resolve the grain structure with approximately $2^{3}, 4^{3}, 8^{3}$, and $16^{3}$ hexahedral elements per grain, respectively. The grain structure begins to emerge at the $r 2$ level of mesh refinement, and is clearly discernible at higher levels of mesh refinement, $r 3$ and $r 4$.

In the interest of modeling as many grains as possible within the macroscale structure, a mesh refinement level is used such that there are approximately $4^{3}$ hexahedral elements per grain (two levels of mesh refinement beyond the first mesh that resolves the grain structure). For the example in Figure 4, this corresponds to the $r 2$ mesh refinement. The effect of higher levels of mesh refinement with respect to the grain size is studied in Appendix A.

Figure 5 shows the number of finite elements required in direct numerical simulation as a function of volume of material for three grain sizes: $100 \mu \mathrm{m}, 50 \mu \mathrm{m}$, and $25 \mu \mathrm{m}$. Figure 5(a) corresponds to the use of $4^{3}$ elements per grain, and Figure 5(b) corresponds to the use of $8^{3}$ elements per grain. For solid mechanics, petascale computing can conceivably process and solve roughly $10^{9}$ elements in a single simulation. From Figure 5(a), this number of elements can simulate $2 \mathrm{~cm}^{3}$ of material for a grain size of $50 \mu \mathrm{m}$ when using $4^{3}$ element per grain. A factor of $10^{3}$ increase in computing performance, known as exascale computing, is predicted to be available within the decade [3], allowing for the direct numerical simulation of roughly $10^{12}$ elements. From Figure 5(a), this number of elements can simulate over $10^{3} \mathrm{~cm}^{3}$ of material, for a grain size of $50 \mu \mathrm{m}$ using $4^{3}$ elements per grain. These volumes are reduced by a factor of 8 


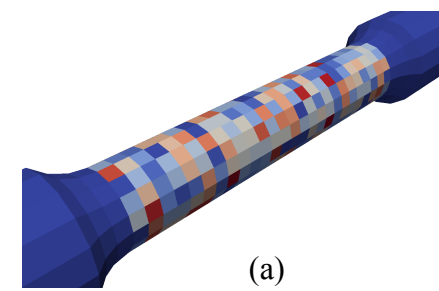

(a)

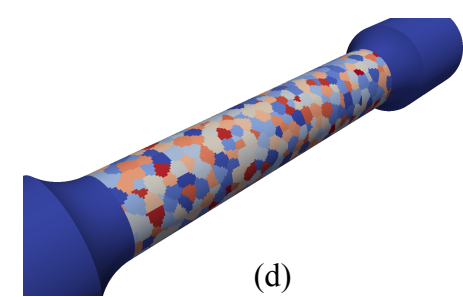

(b)

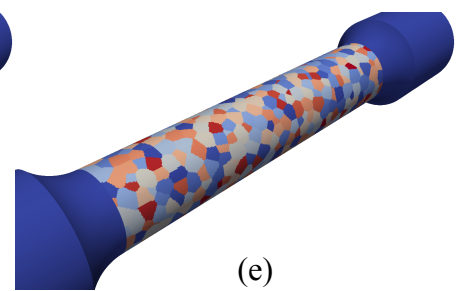

Figure 4: A succession of refined hexahedral meshes illustrating the resolution of the grain structure. There are approximately six grains through the diameter of the gauge section of the round-bar specimen. (a) Base $r 0$ hexahedral mesh with approximately 1 hexahedral element per grain. (b)-(e) Mesh refinements $r 1$ through $r 4$ with approximately $2^{3}, 4^{3}$, $8^{3}$, and $16^{3}$ hexahedral elements per grain, respectively. The relative mesh refinement shown in case (c) is used for all results presented in this paper.

when using $8^{3}$ elements per grain.

\section{Material and microstructural model}

The example polycrystalline material used in this study is AISI 304L stainless steel. This material possesses an austenitic $(\gamma$-Fe) microstructure. A micrograph is shown in Figure 6(a). The FCC crystal structure possesses cubic elastic symmetry with a relatively large anisotropy ratio, $A$, with $A=2 C_{12} /\left(C_{11}-C_{44}\right)=3.5$, where $C_{11}, C_{12}$, and $C_{44}$ are the cubic elastic constants. For an isotropic material, $A=1$. The elastic constants are given in Table 1 for 304L [32] along with those of several other cubic metals for comparison [33].

\subsection{Microstructural model}

The stainless steel microstructure is idealized as a Voronoi tessellation seeded using a Poisson process as demonstrated in Figure 6(b)-(d). Given a finite set of points $\left\{\boldsymbol{x}_{i}\right\}$, called nuclei or seeds, the Voronoi tessellation is defined as the collection of regions or cells $V_{i}$, where

$$
V_{i}=\bigcap_{i \neq j}\left\{\boldsymbol{x} \mid d\left(\boldsymbol{x}_{i}, \boldsymbol{x}\right)<d\left(\boldsymbol{x}_{j}, \boldsymbol{x}\right)\right\}
$$



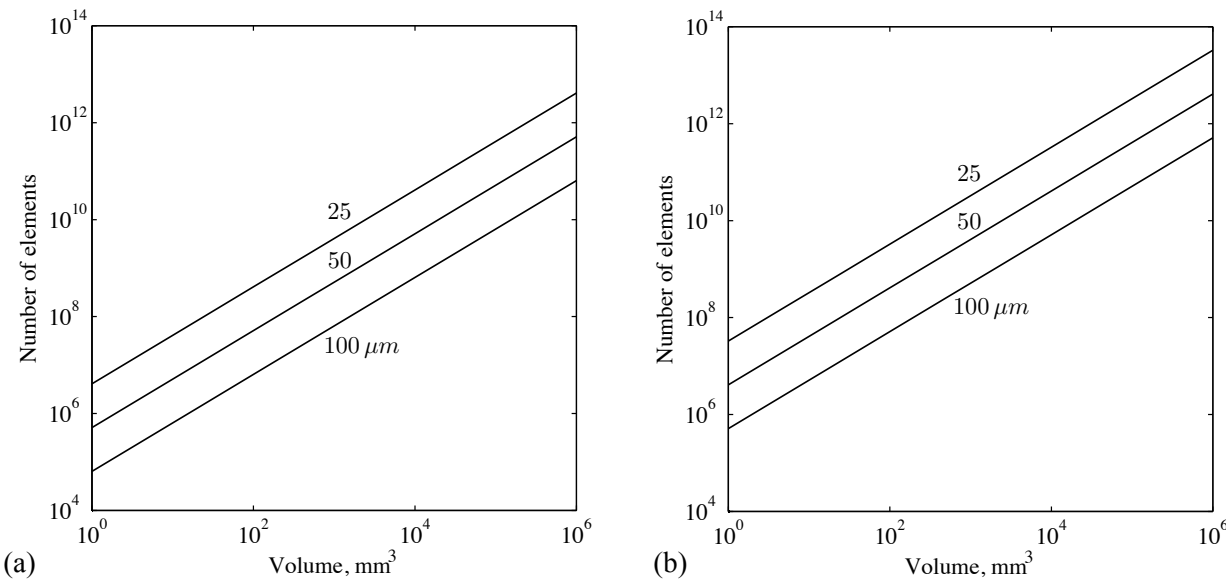

Figure 5: Number of finite elements required in a direct numerical simulation as a function of volume of material for several grain sizes and numbers of elements per grain: (a) $4^{3}$ elements per grain, and (b) $8^{3}$ elements per grain.

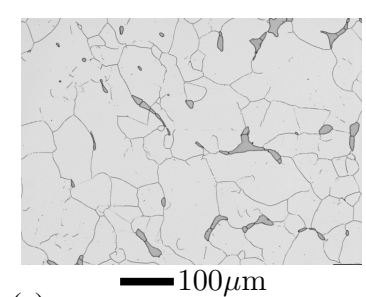

(a)

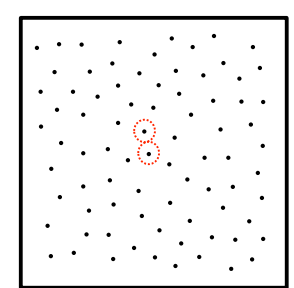

(b)

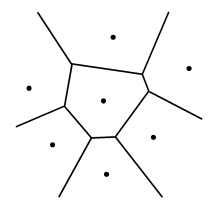

(c)

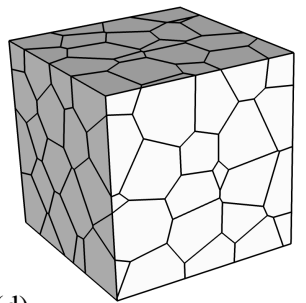

(d)

Figure 6: (a) Micrograph of 304L VAR stainless steel (courtesy Helena Jin, Sandia National Laboratories [34]). (b) Random close-packing of a 2D region also known as maximal Poisson disk sampling (MPS). Two exclusion disks are identified. (c) Voronoi diagram of a set of points in the plane. (d) Voronoi tessellation of a 3D region after MPS sampling.

Table 1: Cubic elasticity constants for 304L stainless steel [32] (units are GPa). The anisotropy ratio $A=2 C_{12} /\left(C_{11}-C_{44}\right)$ is also given. For an isotropic material, $A=1$. Several other cubic metals are given for comparison [33].

\begin{tabular}{ccccc}
\hline material & $C_{11}$ & $C_{12}$ & $C_{44}$ & $\mathrm{~A}$ \\
\hline $304 \mathrm{~L}$ & 204.6 & 137.7 & 126.2 & 3.5 \\
$\alpha-\mathrm{Fe}$ & 231.4 & 134.7 & 116.4 & 2.4 \\
$\mathrm{Al}$ & 107.3 & 60.9 & 28.3 & 1.2 \\
$\mathrm{Cu}$ & 168.4 & 121.4 & 75.4 & 3.2 \\
\hline
\end{tabular}


Here, $\boldsymbol{x}$ represents an arbitrary point in the domain, and the function $d(\boldsymbol{x}, \boldsymbol{y})$ is the Euclidean distance between points $\boldsymbol{x}$ and $\boldsymbol{y}$. Each spatial point belonging to the Voronoi cell $i$ is closer to nucleus $i$ than all other nuclei. Note that each Voronoi cell is defined as the intersection of half-spaces and is thus a convex polyhedron, except possibly at the boundary of a domain. Each Voronoi polyhedron has linear edges and planar faces. Each cell of the Voronoi tessellation is identified as a grain. While only an approximation to the true microstructure (e.g., the faces of each Voronoi cell are planar while the surfaces of each grain are generally non-planar), in the present study we are not interested in the fine scale detail near the grain boundaries. The direct numerical simulation approach presented in Section 3 uses a voxelation approach. The level of fidelity of the voxelation discretization of the grains is consistent with the idealization of the grains using Voronoi structures.

While the Voronoi tessellation can be formed from any finite set of points or seeds, a special structure arises from the close packing of equisized hard spheres, called random close-packing (RCP) [19]. The RCP structure is realized by a type of spatial sampling process known as maximal Poisson disk sampling (MPS). Points are sampled from the multidimensional uniform distribution, and each sample is sequentially placed in the domain with a constraint on minimum distance between points. This constraint is enforced by merely discarding those new points that violate the constraint. The seeding process stops when the maximum packing threshold is reached within tolerance as shown in Figure 6(b). This grain seeding process is repeated using a new seed for the random number generator for each microstructural realization. An efficient method for generating the MPS seeding was developed by Ebeida and Mitchell [35], and is used in this work. The statistical properties of RCP structures have been studied by Finney [36]. In three dimensions the average number of nearest neighbors is 14.3. For comparison, the number of nearest neighbors of the hexagonal close-packed structure is exactly 14 . For the RCP structure, the average aspect ratio of each Voronoi cell is approximately one. Thus, the RCP Voronoi structure provides an equiaxed grain structure.

The crystal orientation is taken to be constant within each Voronoi cell, or grain. The orientation of each grain is taken to be an independent, identically distributed, random variable with no preferred orientation (no texture). This microstructure inherently has a correlation length that is less than the mean grain size. Because of the absence of crystal texture, the homogenized elastic response of the material is isotropic. Care is needed in randomly assigning the grain orientations 
to actually realize a no-texture state [37]. The effective isotropic properties will be obtained in Section 4.2

The identification of vertices, edges, and faces of the Voronoi structure is not explicitly needed in the finite-element modeling (DNS) described in Section 3. Only the identification of a finite element centroid within a particular Voronoi cell (grain) using Eq. (5) is necessary. Thus, the Voronoi model of grain structures is very efficient. Also, note that a type of non-equiaxed grain structure could be created by directionally scaling the final Voronoi structure.

\subsection{Effective material properties}

By construction, the model microstructure defined in Section 4.1 is homogeneous and isotropic at the macroscale. If the microstructure were periodic, periodic boundary conditions could be applied to a unit cell to calculate the homogenized material properties following the standard asymptotic homogenization procedure [9, 12, 22, 23]. For the present random polycrystalline microstructure, in which a periodic unit cell does not exist, the homogenized material properties are obtained from a limiting process in which increasingly larger sample sizes are considered. This limiting process is necessary to obtain unique material properties that are not dependent upon the boundary conditions applied to the sample of material.

Consider a sequence of increasingly larger material volumes (samples), and thus an increasing number of grains, each with geometric similitude. Each sample volume and microstructural realization is called a stochastic volume element (SVE) [38], since the recovered material properties are inherently stochastic. Only in the limit of an infinitely large SVE do these apparent material properties become independent of the boundary conditions applied to the SVE (e.g., statically uniform tractions, kinematically uniform displacements, or periodic displacements). The resulting unique material properties are called effective material properties. These properties are deterministic. Any SVE of finite size, but sufficiently large with apparent material properties approximately equal to the effective material properties, is called a representative volume element (RVE). The appropriate RVE size and the convergence behavior of the apparent material properties to the effective material properties have been studied extensively in the literature $[18,39,40,41,42,26]$.

The terms apparent and effective material properties were defined by Huet [43] in his proof of the Partition Theorem. In his development of the theorem, Huet considered partitions of an RVE into a hierarchy of increasingly smaller subvolumes (SVEs). By considering the stiffness 
tensor of each subvolume of the partition, and then averaging these tensors, Huet was able to develop a partial-order relation [44] of the apparent stiffness tensors of the SVE hierarchy [43],

$$
\mathbb{C}_{\sigma, L}^{\mathrm{app}} \leq \mathbb{C}_{\sigma, 2 L}^{\mathrm{app}} \leq \mathbb{C}_{\sigma, 4 L}^{\mathrm{app}} \leq \cdots \leq \mathbb{C}_{\sigma, \infty}^{\mathrm{app}}=\mathbb{C}_{\varepsilon, \infty}^{\mathrm{app}} \leq \cdots \leq \mathbb{C}_{\varepsilon, 4 L}^{\mathrm{app}} \leq \mathbb{C}_{\varepsilon, 2 L}^{\mathrm{app}} \leq \mathbb{C}_{\varepsilon, L}^{\mathrm{app}},
$$

where $\mathbb{C}_{\sigma, \infty}^{\text {app }}=\mathbb{C}_{\varepsilon, \infty}^{\text {app }} \equiv \mathbb{C}^{\text {eff }}$ is the effective stiffness tensor. Here, the subscript $L$ denotes the dimension of the smallest considered SVE. The subscripts $\sigma$ and $\varepsilon$ denote the use of statically uniform and kinematically uniform boundary conditions, respectively. This partial-order relation is understood in an energetic sense such that $\mathbb{C}_{1} \leq \mathbb{C}_{2}$ if and only if $\varepsilon:\left(\mathbb{C}_{2}-\mathbb{C}_{1}\right): \varepsilon \geq 0$ for all strain tensors $\boldsymbol{\varepsilon}$.

To obtain the effective material properties for the model polycrystalline microstructure given in Section 4, a hierarchy of SVE volumes is considered. The smallest volume contains approximately $4^{3}$ grains. Subsequent volumes are obtained by doubling the dimensions resulting in 8 times the volume and approximately 8 times as many grains as the previous SVE size. A total of 4 volumes are considered in the sequence with the largest volume containing approximately $32^{3}$ grains. The three largest SVE volumes are shown in Figure 7. Three microstructural realizations are shown for each volume size. For the finite-element discretization of each SVE, the voxelation paradigm described in Section 3 is used. The smallest volume is meshed uniformly with $16^{3}$ elements. There are 8 times as many finite elements for each subsequent volume in the sequence.

Statically uniform tractions, both shear and normal stress, are applied to the faces of each SVE to recover the full apparent compliance tensor, $\mathbb{S}_{\sigma}^{\text {app }}$. Kinematically uniform or periodic displacement boundary conditions could have been used as well. The Von Mises stress fields for the SVEs shown in Figure 7 (first realization) resulting from a uniaxial tension case are shown in Figure 8. The compliance tensor is inverted to obtain the apparent stiffness tensor, $\mathbb{C}_{\sigma}^{\mathrm{app}}$. Both $\mathbb{S}_{\sigma}^{\mathrm{app}}$ and $\mathbb{C}_{\sigma}^{\mathrm{app}}$ are random and generally anisotropic. However, as the sample volume increases and the number of grains increases, the deviation from isotropy diminishes. Additionally, deviations from the ensemble mean behavior are increasingly rare. This is analogous to the behavior of a random variable obtained from the sum of independent identically distributed random variables as described by the central limit theorem [45], and illustrates the motivation behind homogenization theory.

For each SVE size, an ensemble consisting of 100 realizations of the model microstructure is analyzed. The ensemble-averaged apparent stiffness tensor is then projected to the closest 
(a)
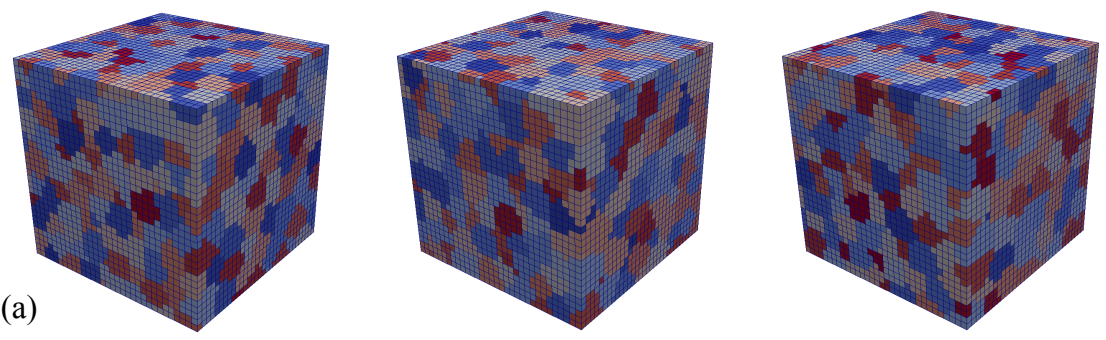

(b)
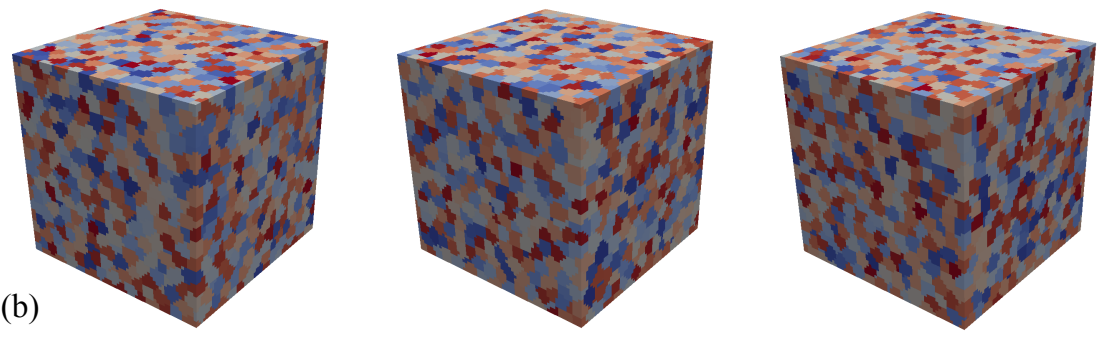

(c)
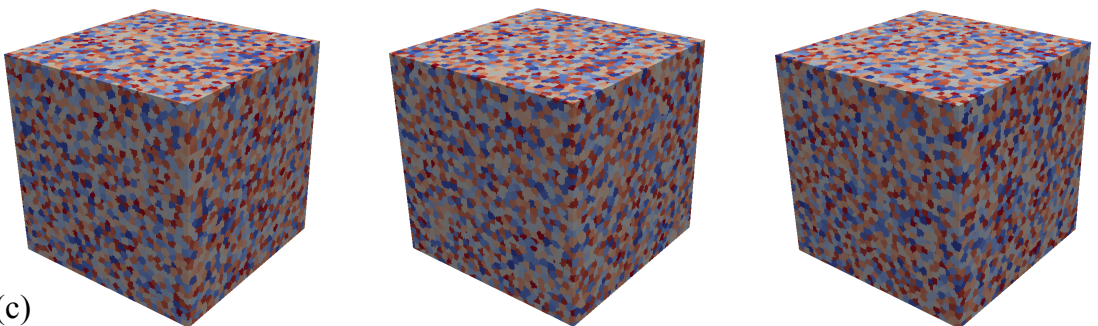

Figure 7: Stochastic volume elements (SVE) of increasing size and thus with increasing numbers of grains. Three realizations are shown for each SVE size: (a) approximately $8^{3}$ grains per realization, (b) approximately $16^{3}$ grains per realization, and (c) approximately $32^{3}$ grains per realization. The apparent anisotropic compliance tensor is recovered for each SVE and used to obtain the effective isotropic properties given in Table 2.
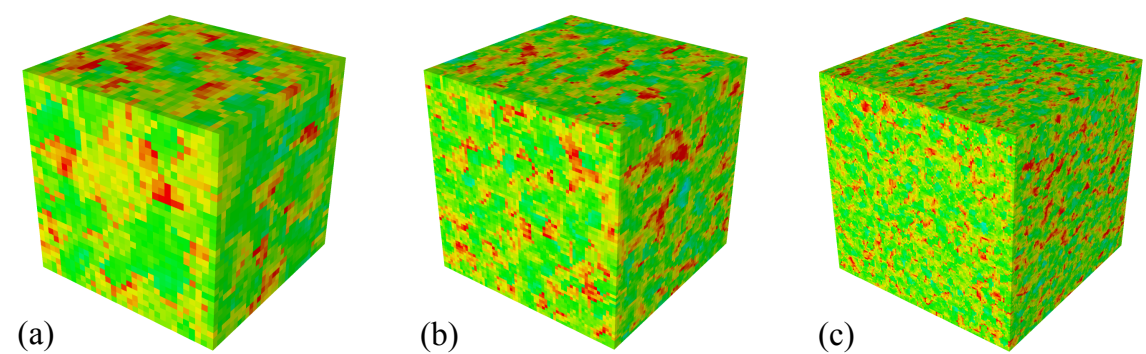

Figure 8: Von Mises stress field for the SVEs shown in Figure 7 (first realization) resulting from a uniaxial tension load case: (a) approximately $8^{3}$ grains per realization, (b) approximately $16^{3}$ grains per realization, and (c) approximately $32^{3}$ grains per realization. 
isotropic stiffness tensor in the space of symmetric fourth-order stiffness tensors using the logEuclidean metric (distance function) [46],

$$
d_{L}\left(\mathbb{C}_{1}, \mathbb{C}_{2}\right)=\left\|\log \left(\mathbb{C}_{1}\right)-\log \left(\mathbb{C}_{2}\right)\right\|
$$

where $\|\mathbb{A}\|=\sqrt{A_{i j p q} A_{p q i j}}$. This distance function has the desirable properties of being invariant under inversion and positive-scalar multiplication,

$$
d_{L}\left(\mathbb{C}_{1}, \mathbb{C}_{2}\right)=d_{L}\left(\mathbb{S}_{1}, \mathbb{S}_{2}\right) \quad \text { and } \quad d_{L}\left(a \mathbb{C}_{1}, a \mathbb{C}_{2}\right)=d_{L}\left(\mathbb{C}_{1}, \mathbb{C}_{2}\right), \quad a>0
$$

Moakher and Norris [46] derived explicit formulas for the closest isotropic stiffness tensor using the log-Euclidean distance function. First, a general isotropic stiffness tensor can be written as

$$
\mathbb{C}_{\text {iso }}=3 K \mathbb{J}+2 \mu \mathbb{K}
$$

where $K$ and $\mu$ are scalars, and $\mathbb{J}$ and $\mathbb{K}$ are two linearly independent symmetric tensors defined by $J_{i j k l}=\frac{1}{3} \delta_{i j} \delta_{k l}$ and $\mathbb{K}=\mathbb{I}-\mathbb{J}$ with $I_{i j k l}=\frac{1}{2}\left(\delta_{i k} \delta_{j l}+\delta_{i l} \delta_{j k}\right)$. For a general anisotropic stiffness tensor, the moduli $K_{L}$ and $\mu_{L}$ of the closest isotropic stiffness tensor are given by

$$
3 K_{L}=\exp (\operatorname{tr}(\mathbb{J} \log (\mathbb{C}))) \quad 2 \mu_{L}=\exp \left(\frac{1}{5} \operatorname{tr}(\mathbb{K} \log (\mathbb{C}))\right)
$$

where $\operatorname{tr}(\cdot)$ is the trace operator.

The Young's modulus and Poisson's ratio are obtained using the standard conversion formulae for an isotropic material. Results for each SVE volume are given in Table 2. These apparent material properties are then extrapolated to an infinite SVE volume (or, equivalently, an infinitesimally small grain size) using Richardson extrapolation [47] to obtain the effective material properties. These values are given in the last row of Table 2. The effective material properties are consistent with measured stainless steel material properties given by Ledbetter, $e t$ al. [48]. Interestingly, the derived convergence rate from the Richardson extrapolation procedure is approximately one, so that a doubling of the dimensions of the SVE only reduce the error in the apparent material properties by approximately a factor of 0.5 .

\section{Direct numerical simulations of an I-beam}

The example macroscale structure is an I-beam with holes in the web region and fillets connecting the web and flange as shown in Figure 9. The I-beam is quasi-statically loaded in a 


Table 2: Convergence of the ensemble-averaged apparent isotropic material properties to the effe
properties. Richardson extrapolation [47] is used to obtain the effective material properties in the
grains goes to infinity (last row).
\begin{tabular}{ccc}
\hline $\begin{array}{l}\text { approximate } \\
\text { number of grains }\end{array}$ & $\begin{array}{l}\text { apparent Young's } \\
\text { modulus (GPa) }\end{array}$ & $\begin{array}{c}\text { apparent } \\
\text { Poisson's ratio }\end{array}$ \\
\hline $4^{3}$ & 185.2 & 0.307 \\
$8^{3}$ & 190.5 & 0.301 \\
$16^{3}$ & 193.9 & 0.298 \\
$32^{3}$ & 195.7 & 0.296 \\
\hline$\infty$ & 197.6 & 0.294 \\
\hline
\end{tabular}

torsional mode to create stress gradients throughout the structure along with active stress concentrations in the web region. (These stress gradients and stress concentrations are less evident for other types of loadings, including bending.) The DNS simulations of the I-beam are discussed as follows. The dimensions of the I-beam and definition of the torsional loading are given in Section 5.1. Details of the hexahedral meshing are given in Section 5.2. The embedded microstructure is shown in Section 5.3 for two grain sizes. Displacement and stress results are given in Section 5.4 for two realizations of each grain size. These results are then compared to the displacement and stress fields obtained using the homogenized (effective) material properties from Section 4.2. The statistics of an ensemble of 100 DNS simulations is studied in Section 5.5. Finally, Section 5.6 discusses spatially filtered stress results.

\subsection{I-beam geometry and loading}

The dimensions of the I-beam are given in Figure 10 in nondimensional units. For specificity, we take the units to be millimeters, but only the size of the beam relative to the size of the grain structure is significant. This ratio will be denoted throughout as the "web thickness-to-grain ratio." Thus, the I-beam is $40 \mathrm{~mm}$ long and the web-section thickness is $1 \mathrm{~mm}$. The volume of the I-beam is approximately $970 \mathrm{~mm}^{3}$.

To apply torsion loading, equal and opposite surface tractions are applied at both end-surfaces 


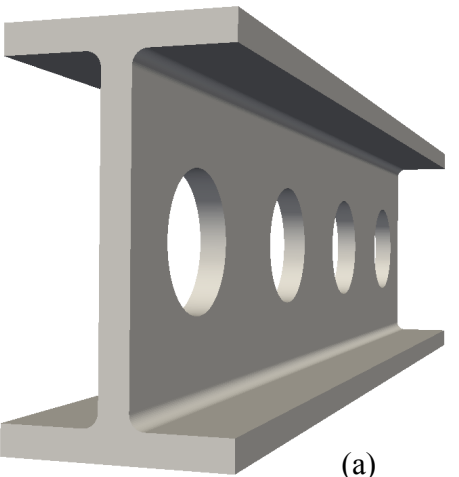

(a)

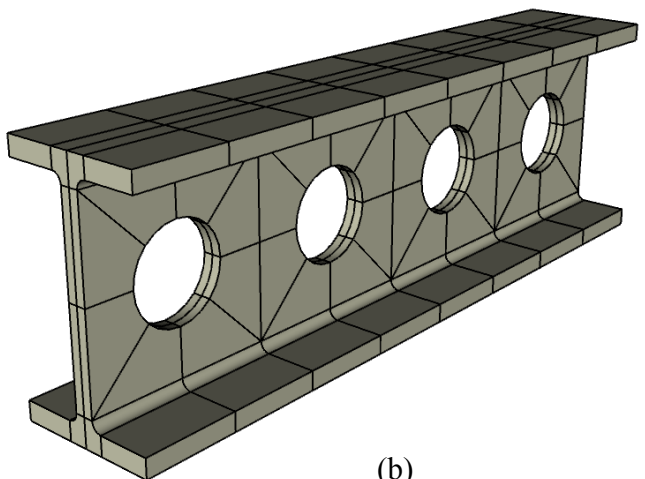

(b)

Figure 9: (a) Model macroscale structure, an I-beam. (b) Decomposition of the I-beam into subregions for hexahedral meshing.

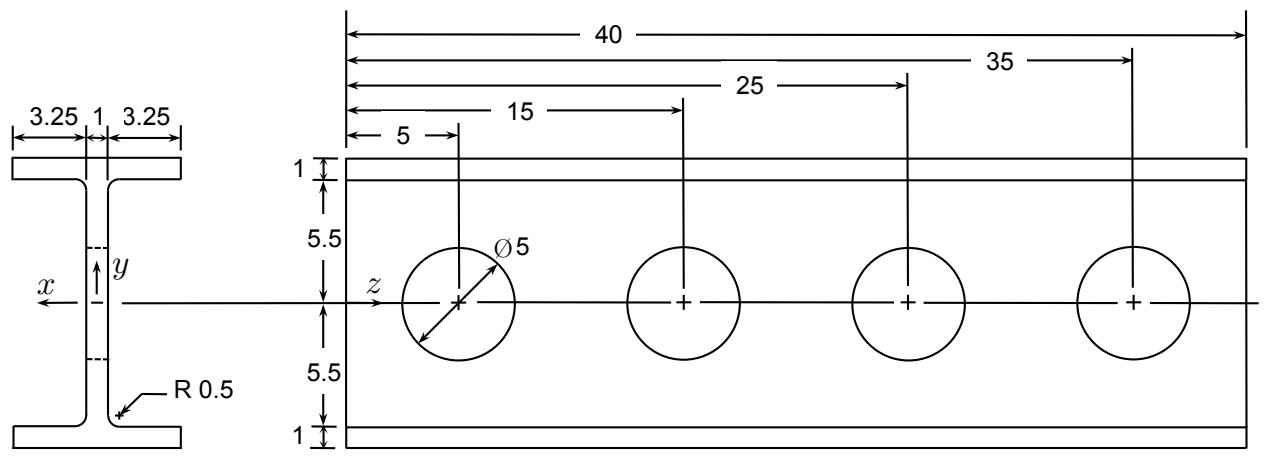

Figure 10: Dimensions of the example macroscale structure, an I-beam with holes in the web section. A rectilinear coordinate system, $x y z$, is identified. 
of the I-beam $(z=0$ and $z=40)$. The traction vectors are given by

$$
\left.\left.\begin{array}{c}
T_{x}=-c(r \sin \theta) \\
T_{y}=-c(r \cos \theta) \\
T_{z}=0
\end{array}\right\} \text { at } z=0 \text { and } \begin{array}{c}
T_{x}=c(r \sin \theta) \\
T_{y}=c(r \cos \theta) \\
T_{z}=0
\end{array}\right\} \quad \text { at } z=40,
$$

where $r^{2}=x^{2}+y^{2}, \theta=\arctan (y / x)$, and $c$ is a scale factor taken to be $1 / 3$. Rigid-body motion is removed by specifying six displacement constraints using three points on the plane $z=0$ located at extremal vertices of the cross-section: point $1(x=3.75, y=-6.5, z=0)$, point 2 $(x=-3.75, y=-6.5, z=0)$, point $3(x=3.75, y=6.5, z=0)$. Point 1 is fixed in all three coordinate directions. Point 2 is constrained in the $y$ and $z$ coordinate directions while point 3 is constrained only in the $z$ coordinate direction.

\subsection{I-beam meshing}

The meshing software CUBIT [31] is used to create both the geometry and mesh of the Ibeam. In order to mesh the I-beam with hexahedra, it is first partitioned into geometric hexahedra as shown in Figure 9(b). The multiple symmetry plains of the I-beam are exploited so that only three subregions need to be meshed. The remaining regions are meshed through simple reflections and translations. The base hexahedral mesh of the I-beam is shown in Figure 11(a). This base mesh contains 8576 hexahedra, and contains two hexahedra through the web and flange thicknesses. Subsequent hexahedral meshes, $r 1, r 2, r 3, \ldots$ are obtained from uniform hierarchical refinement in which each hexahedron is divided into eight. New surface nodes created during each refinement are located on the exact geometry. The $r 1$ mesh refinement is shown in Figure 11(b) and contains 68608 hexahedra. The mesh refinements $r 2, r 3, r 4, r 5$ contain approximately 549 thousand, 4.39 million, 35.1 million, and 281 million hexahedra, respectively. The $r 3$ and $r 4$ mesh refinements are used in the following simulations.

\subsection{Embedded microstructure}

The model microstructure defined in Section 4 is embedded into the I-beam macroscale structure using the DNS methodology described in Section 3. Our goal is to investigate the error in homogenization theory for finite microstructures. We start with a grain size resulting in approximately 4 grains through the web-section thickness using the $r 3$ hexahedral mesh refinement. For a web-section thickness of $1 \mathrm{~mm}$, this implies that the grain size is approximately $250 \mu \mathrm{m}$. A 
Figure 11: The base hexahedral mesh of the I-beam, denoted as $r 0$, contains 8,576 hexahedra. Subsequent hexahedral meshes, $r 1, r 2, r 3, \ldots$ are obtained from uniform hierarchical refinement in which each hexahedral element is divided into eight elements. New surface nodes created during each refinement are located on the exact geometry. The refinements $r 1, r 2, r 3, r 4$ (not shown) contain approximately 69 thousand, 549 thousand, 4.39 million, 35.1 million hexahedra, respectively.

realization of the microstructure with this grain size is shown in Figure 12. The total number of grains for this realization is approximately 60 thousand. A cross-sectional view through the mid-section of the I-beam $(z=20)$ is also shown. A microstructural realization with approximately 8 grains through the web-section thickness using the $r 4$ mesh refinement is shown in Figure 13. For a web-section thickness of $1 \mathrm{~mm}$, this implies that the grain size is approximately $125 \mu \mathrm{m}$. The total number of grains for this realization is approximately 420 thousand. The case of 16 grains through the web-section thickness using the $r 5$ mesh refinement (approximately 281 million elements) is the subject of an ongoing investigation.

\subsection{Displacement and Stress results}

As described in Section 3, the I-beam boundary-value problem is solved using the solid mechanics module within the Sierra multiphysics finite-element software suite [29] and the FETIDP domain-decomposition iterative solver [30]. For each microstructural realization using the $r 3$ mesh refinement (containing 4.39 million elements), 320 processors were used with typical runtimes of approximately 5 minutes. For each microstructural realization using the $r 4$ mesh refinement (containing 35.1 million elements), 2400 processors were used with typical runtimes 


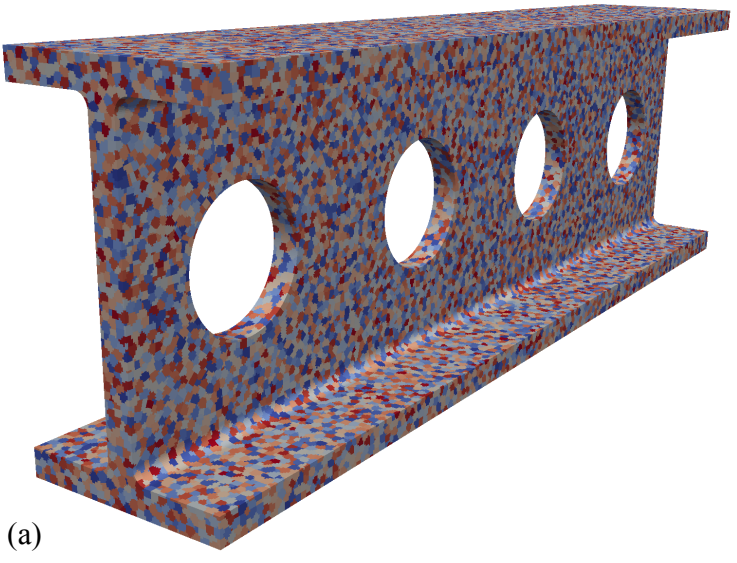

(b)

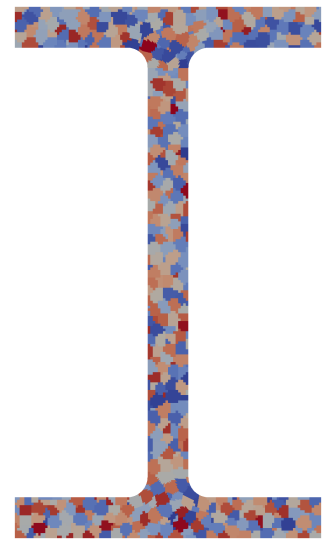

Figure 12: (a) I-beam with embedded microstructure using the $r 3$ hexahedral mesh refinement. There are approximately 4 grains through the web and flange thicknesses, i.e., thickness-to-grain ratio $=4$. The total number of grains for this realization of the microstructure is approximately 60 thousand. (b) A cross-section through the mid-section of the I-beam $(z=20)$.

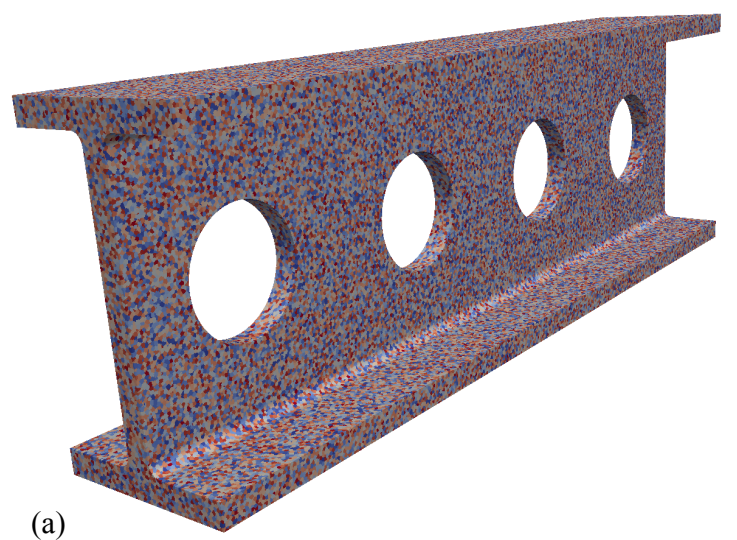

(b)

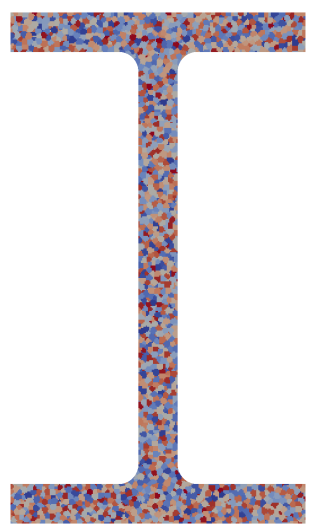

Figure 13: (a) I-beam with embedded microstructure using the $r 4$ hexahedral mesh refinement. There are approximately 8 grains through the web and flange thicknesses, i.e., thickness-to-grain ratio $=8$. The total number of grains for this realization of the microstructure is approximately 420 thousand. (b) A cross-section through the mid-section of the I-beam $(z=20)$. 


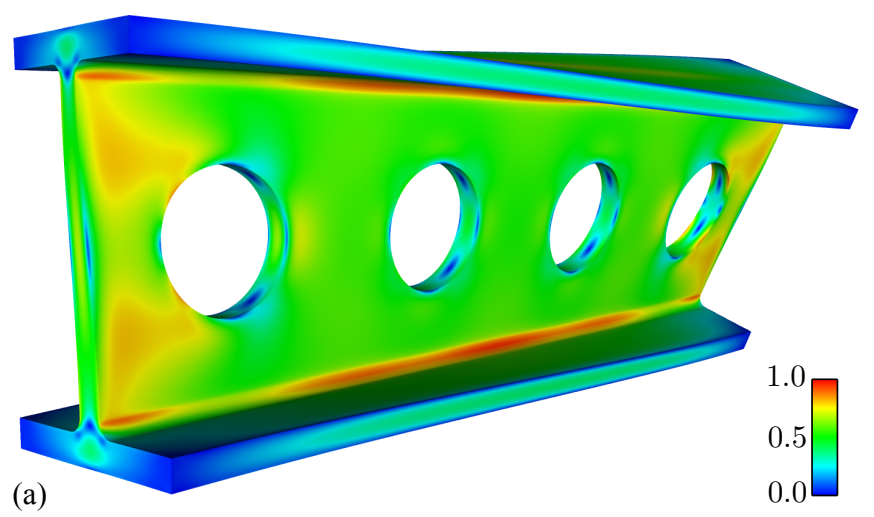

(b)

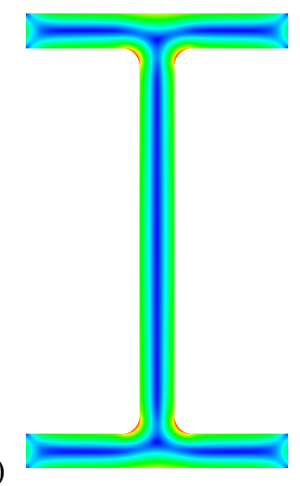

Figure 14: (a) Von Mises stress field resulting from torsional loading using the homogenized isotropic material properties and the $r 4$ mesh refinement. The deformation is shown greatly exaggerated. (b) Cross-sectional view of the Von Mises stress field at the mid-section of the I-beam $(z=20)$. Normalized stress units are used.

of approximately 10 minutes.

The Von Mises stress field resulting from the torsional loading using the homogenized material properties and $r 4$ mesh refinement is shown in Figure 14. The deformation is exaggerated for clarity. A cross-sectional view of the Von Mises stress field at the mid-section of the I-beam $(z=20)$ is also shown. The element-stress values resulting from the finite-element analysis are shown with no smoothing or other post-processing. The smoothness of the stress field is due to the extreme refinement of the finite element mesh. Note that for this loading mode, the stress is concentrated on the surface of the structure. Also, note the stress concentrations in the fillets and around the two end-holes.

The Von Mises stress field resulting from the direct numerical simulation of the I-beam is shown in Figure 15 using the embedded microstructure shown in Figure 12 (web thickness-tograin ratio $=4, r 3$ mesh refinement). A cross-sectional view of the stress field at the mid-section of the I-beam $(z=20)$ is also shown. The DNS stress solution displays large fluctuations on small spatial scales, but is qualitatively similar to the smooth stress field obtained by using the homogenized material properties. These fine-scale fluctuations are effectively filtered out in the homogenized solution as expected. Figure 16 shows the Von Mises stress field resulting from the DNS solution using the embedded microstructure shown in Figure 13 (web thickness-to-grain ratio $=8, r 4$ mesh refinement). Again, the DNS stress field exhibits large spatial fluctuations at fine scales, but is qualitatively similar to the homogenized solution on larger scales. 


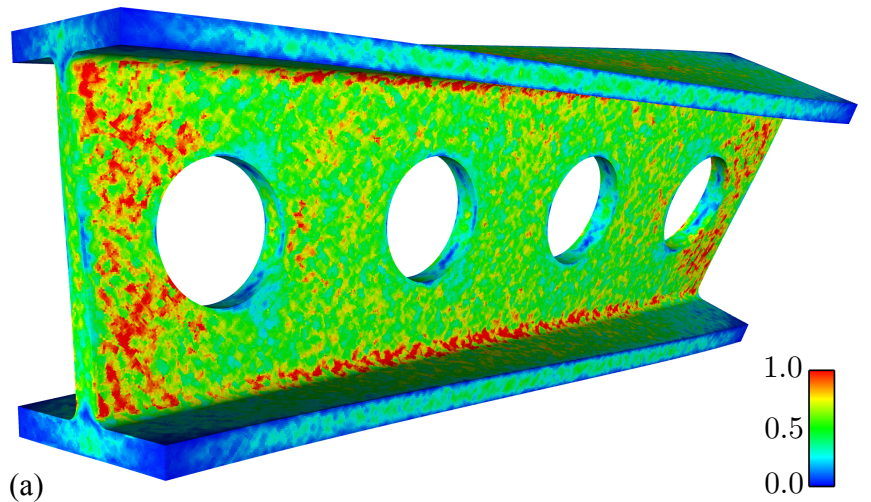

(b)

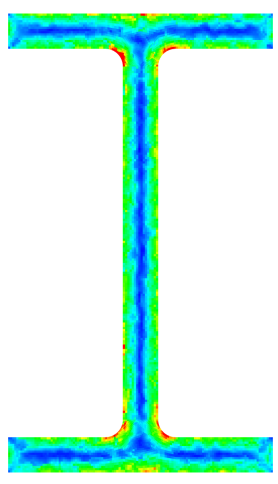

Figure 15: (a) Von Mises stress field resulting from the direct numerical simulation of the microstructure shown in Figure 12 (web thickness-to-grain ratio $=4, r 3$ mesh refinement). (b) Cross-sectional view of the Von Mises stress field at the mid-section of the I-beam $(z=20)$. (cf. Figures 14 and 16.)
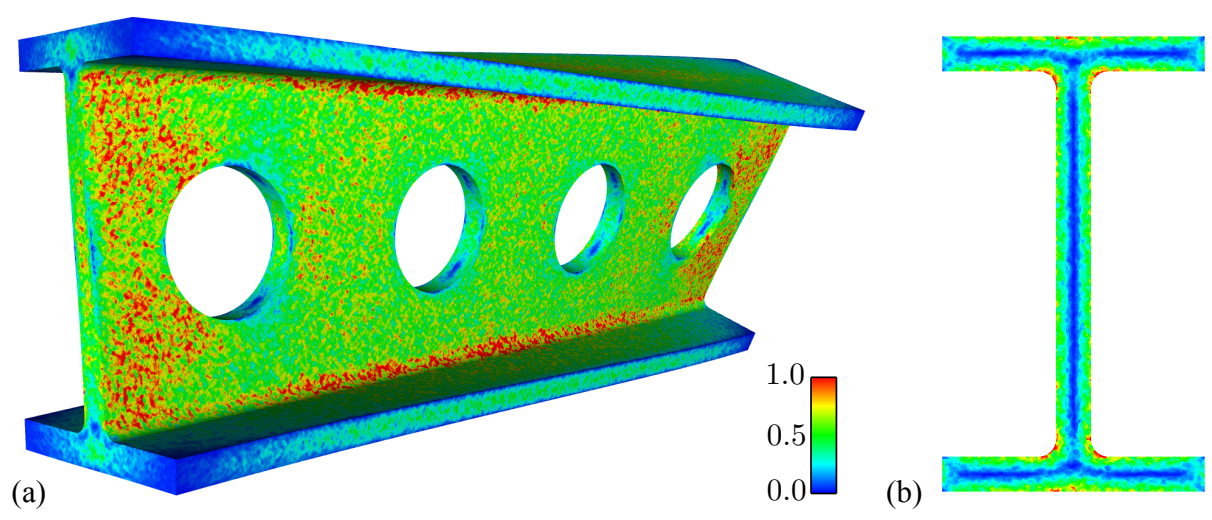

(b)

Figure 16: (a) Von Mises stress field resulting from the direct numerical simulation of the microstructure shown in Figure 12 (web thickness-to-grain ratio $=8, r 4$ mesh refinement). (b) Cross-sectional view of the Von Mises stress field at the mid-section of the I-beam $(z=20)$. (cf. Figures 14 and 15.) 
In order to investigate the differences between the homogenization and DNS solutions, the displacement and stress magnitude along three curves/lines within the I-beam are extracted and graphed in the figures to follow. These curves are identified in Figure 17. Each extraction curve is discretized with 10 thousand points. For each point, the enclosing finite element is found, and a mean element stress is assigned as the stress at the given point. The displacement value at the given point is interpolated from the nodal displacements using the element shape functions. Figure 18 shows the magnitude of the displacement vector along the surface of the web-flange fillet of the I-beam (line 1 identified in Figure 17) for (a) a web thickness-to-grain ratio $=4$ using the $r 3$ mesh refinement, and (b) a web thickness-to-grain ratio $=8$ and using the $r 4$ mesh refinement. Results from two microstructural realizations are shown. These results are nearly indistinguishable and surprisingly smooth. The displacement magnitude resulting from the use of homogenized material properties is also shown. In order to have a valid comparison, the same mesh refinement (either $r 3$ or $r 4$ ) is used for both DNS and homogenization simulations. There is only a small difference between the DNS results and the homogenization results indicating that the overall stiffness of the I-beam is accurately represented by the homogenized material properties. As expected, the difference between the DNS results and the homogenization results decreases when going from a web thickness-to-grain ratio of 4 to a web thickness-to-grain ratio of 8 .

Figure 19 shows the magnitude of the Cauchy stress, $\left(\sigma_{i j} \sigma_{i j}\right)^{1 / 2}$, along the surface of the web-flange fillet of the I-beam (line 1 identified in Figure 17) for (a) a web thickness-to-grain ratio $=4$ using the $r 3$ mesh refinement, and (b) a web thickness-to-grain ratio $=8$ and using the $r 4$ mesh refinement. Results from two microstructural realizations are shown. The first realization uses the microstructure shown in Figure 12. The stress magnitude resulting from the use of homogenized material properties is also shown. Again, no smoothing operations are performed on the stress fields. The smooth stress variation of the homogenization results is due to the extremely fine mesh refinement used in these simulations. Even for these rather large relative grain sizes (only a few grains through the fillet radius), the homogenization results appear to provide a good representation of the spatial mean of the DNS results. Note the rather large local stress in Figure 19(a) at position $z \approx 10$ for realization 1 and at position $z \approx 24$ for realization 2, which is away from the location of the peak stress in the homogenized solution $(z=20)$. Although not the focus of this study, these random fluctuations in the local stress field 

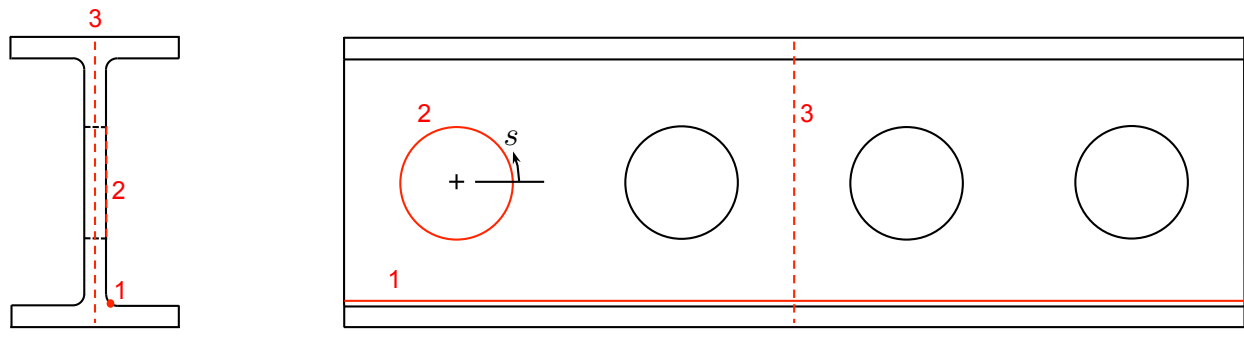

Figure 17: Identification of three lines/curves in the I-beam along which stress results are extracted. Line 1 is along the surface of the web-flange fillet. The coordinates of the begin and end points for line 1 are $(0,-5.355,0.645)$ and $(40,-5.355,0.645)$, respectively. Curve 2 is along the edge of the web-section hole nearest $z=0$. This circle has center $(5,0,0.5)$ and radius 0.25 . A circumferential coordinate, $s$, is also defined. Line 3 runs from the top of the center section to the bottom. The coordinates of the begin and end points for line 3 are $(0,6.5,20)$ and $(0,-6.5,20)$, respectively.

reinforce the importance of microstructural effects in predicting fatigue and fracture initiation. The homogenized solution is perfectly symmetric about the mid-section of the I-beam, whereas the DNS solutions are clearly unsymmetric. Although the stress fluctuations have a greater spatial frequency in Figure 19(b) compared to Figure 19(a), the DNS results are similar. There does not appear to be an offset in the spatially averaged behavior away from the homogenization results for either web thickness-to-grain ratio. The comparison between results of homogenization and direct numerical simulation will be explored further in Section 5.5 when ensemble-averaged results are presented, and in Section 5.6 when a spatial filter is used to filter the DNS results.

Analogous results to those shown in Figures 18 and 19, but around the edge of the hole at the end of the I-beam (curve 2 identified in Figure 17), are shown in Figures 20 and 21, respectively. The displacement results are similar to those shown in Figure 18. The piecewise-constant stress field from the finite-element solution using the $r 3$ mesh refinement is evident in the homogenization results shown in Figure 21(a). This effect is less evident in the $r 4$ mesh refinement used in Figure 21(b). The homogenized solution is perfectly symmetric, whereas the DNS solutions are clearly unsymmetric. In Figure 21(b), there appears to be an offset in the spatial-mean behavior of the DNS results around the locations of the two larger stress peaks in the homogenized solution, at least for these two microstructural realizations. This will be explored further in the ensemble-averaged results presented in Section 5.5 and the spatially averaged results presented in Section 5.6.

To explore the stress results in the interior of the I-beam, the displacement and stress mag- 
(a)

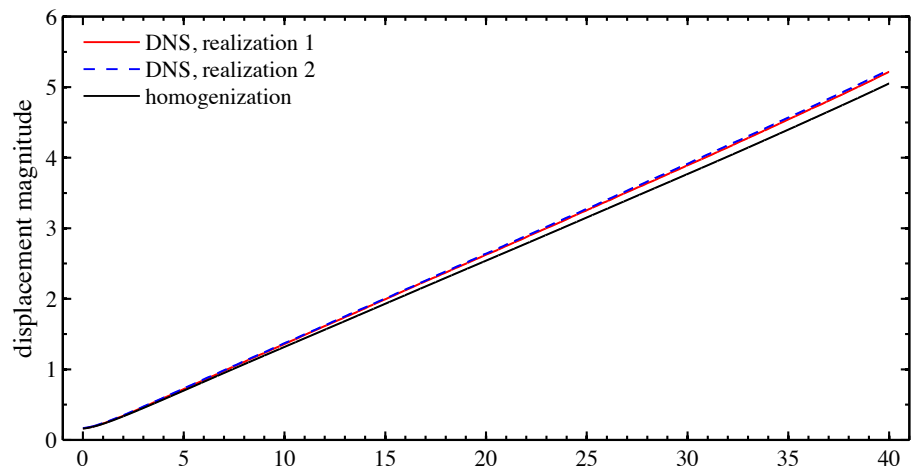

(b)

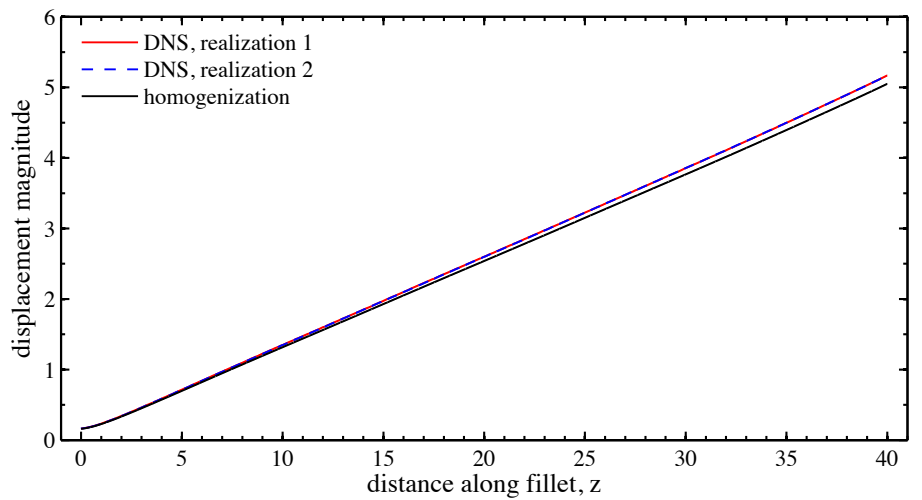

Figure 18: Displacement magnitude (scaled by a factor of $10^{4}$ ) along the surface of the web-flange fillet of the I-beam (line 1 identified in Figure 17) for (a) a web thickness-to-grain ratio $=4$ using the $r 3$ mesh refinement, and (b) a web thickness-to-grain ratio $=8$ and using the $r 4$ mesh refinement. Results from two microstructural realizations are shown. Results obtained using the homogenized material properties are also shown. 
(a)

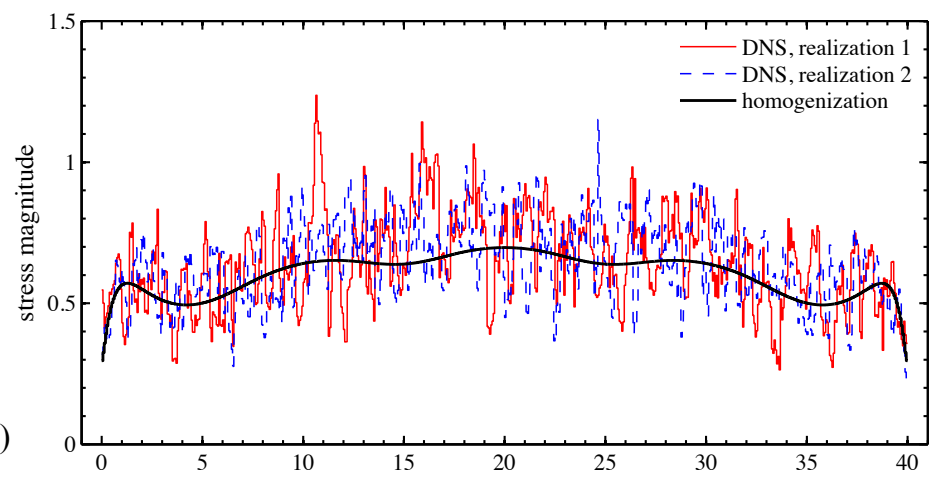

(b)

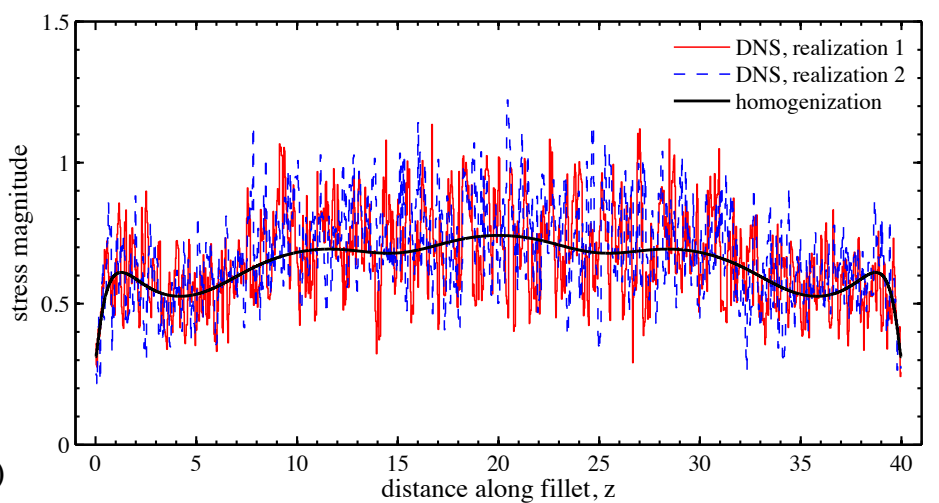

Figure 19: Stress magnitude, $\left(\sigma_{i j} \sigma_{i j}\right)^{1 / 2}$, along the surface of the web-flange fillet of the I-beam (line 1 identified in Figure 17) for (a) a web thickness-to-grain ratio $=4$ using the $r 3$ mesh refinement, and (b) a web thickness-to-grain ratio $=8$ and using the $r 4$ mesh refinement. Results from two microstructural realizations are shown. Results obtained using the homogenized material properties are also shown. 
(a)

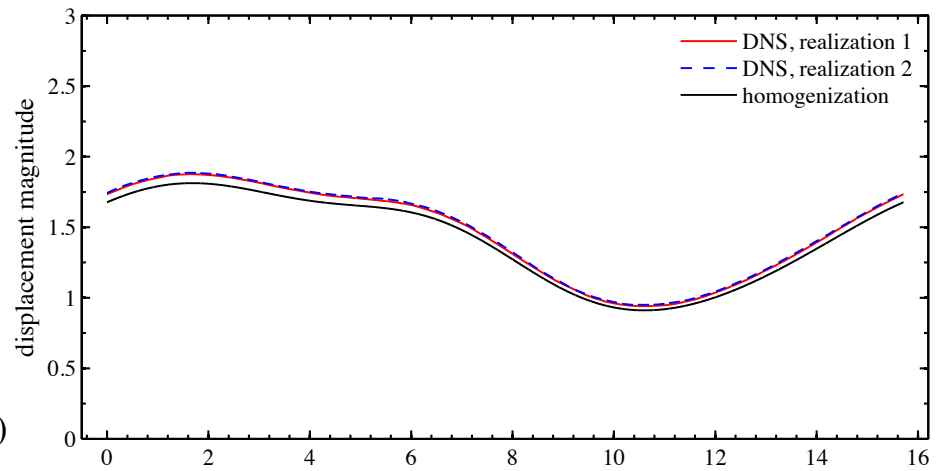

(b)

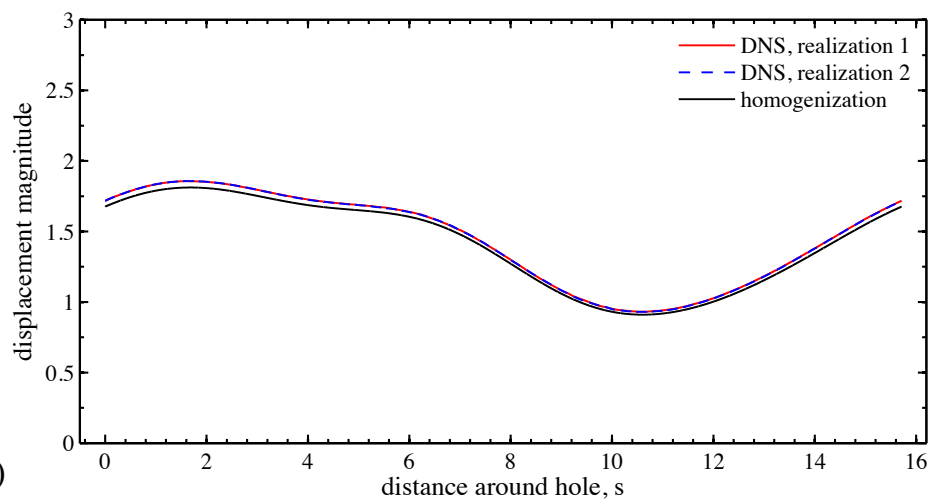

Figure 20: Displacement magnitude (scaled by a factor of $10^{4}$ ) along the edge of the hole at the end of the I-beam (curve 2 identified in Figure 17) for (a) a web thickness-to-grain ratio $=4$ using the $r 3$ mesh refinement, and (b) a web thickness-to-grain ratio $=8$ and using the $r 4$ mesh refinement. Results from two microstructural realizations are shown. Results obtained using the homogenized material properties are also shown. 
(a)

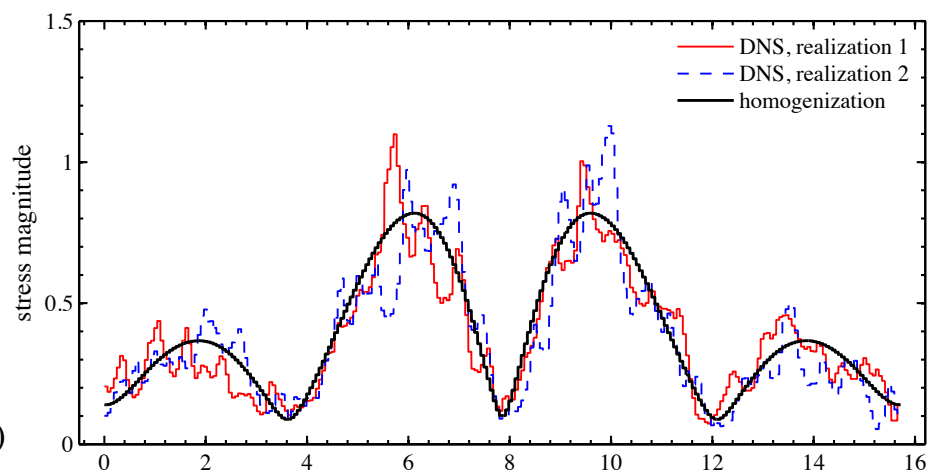

(b)

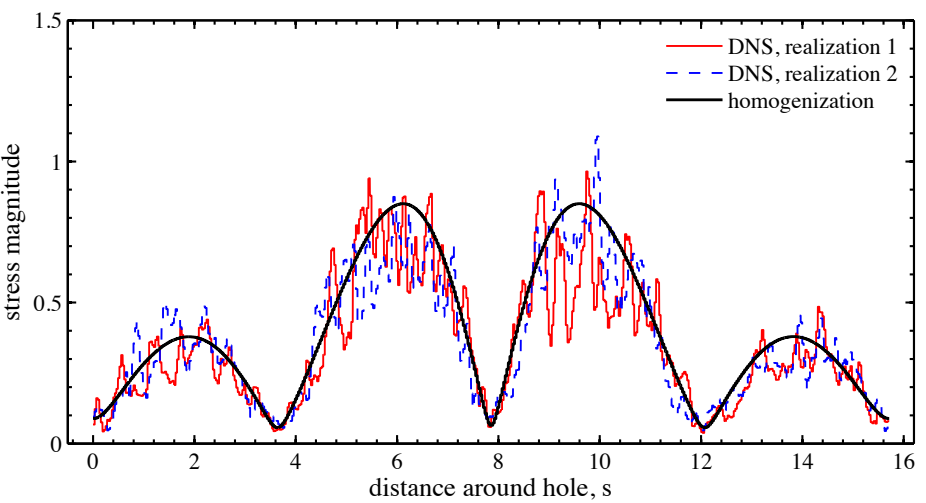

Figure 21: Stress magnitude, $\left(\sigma_{i j} \sigma_{i j}\right)^{1 / 2}$, along the edge of the hole at the end of the I-beam (curve 2 identified in Figure 17) for (a) a web thickness-to-grain ratio $=4$ using the $r 3$ mesh refinement, and (b) a web thickness-to-grain ratio $=8$ and using the $r 4$ mesh refinement. Results from two microstructural realizations are shown. Results obtained using the homogenized material properties are also shown. 
(a)

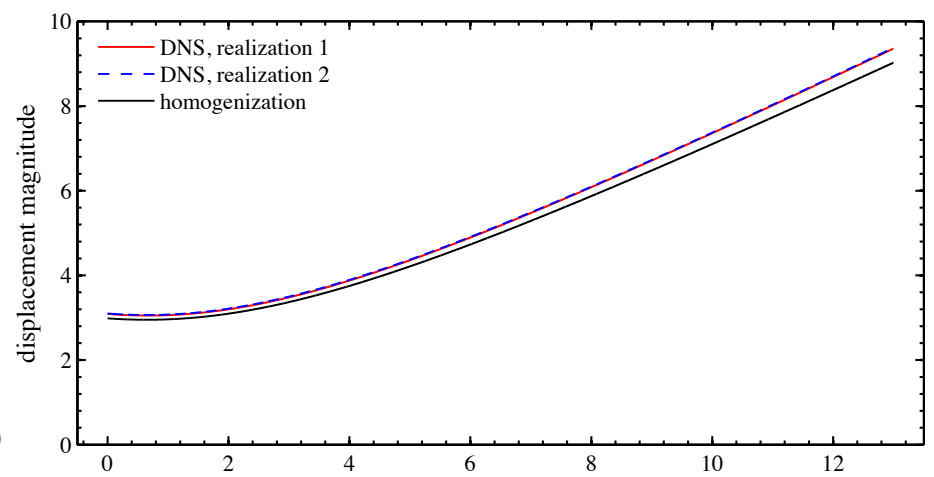

(b)

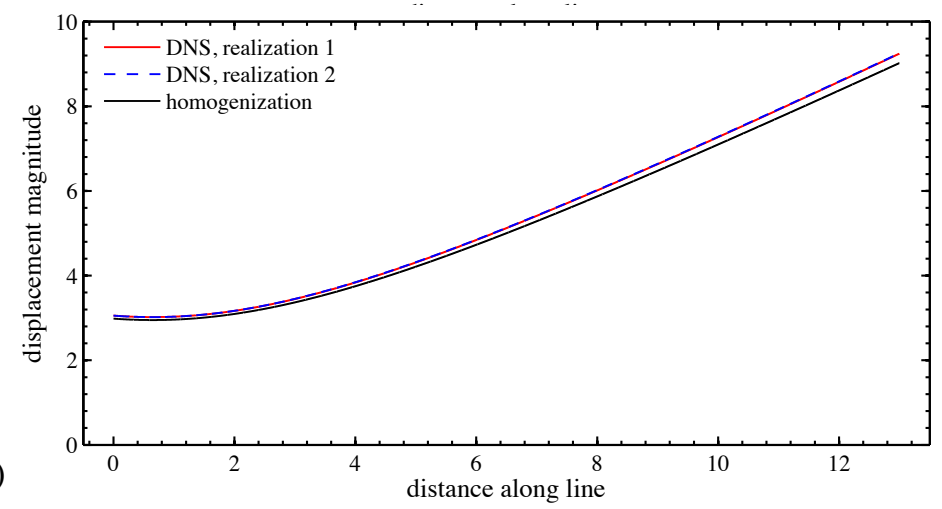

Figure 22: Displacement magnitude (scaled by a factor of $10^{4}$ ) along a line through the center of the I-beam (line 3 identified in Figure 17) for (a) a web thickness-to-grain ratio $=4$ using the $r 3$ mesh refinement, and (b) a web thicknessto-grain ratio $=8$ and using the $r 4$ mesh refinement. Results from two microstructural realizations are shown. Results obtained using the homogenized material properties are also shown.

nitudes are extracted along a line through the center of the I-beam from top to bottom at the mid-section (line 3 identified in Figure 17). These results are shown in Figures 22 and 23. The displacement results are similar to those shown in Figures 18 and 20. For the stress results there is only a small fluctuation of the DNS results about the homogenized solution. One exception is the second microstructural realization shown in Figure 23(b). For this case there is an unusually large stress value in the DNS result at the end point. This location corresponds to the surface of the I-beam flange.

\subsection{Ensemble statistics}

Beran and McCoy [17] and Drugan and Willis [18] have shown that the governing field equations for the ensemble-averaged stress field are nonlocal in character with the nonlocality 
(a)

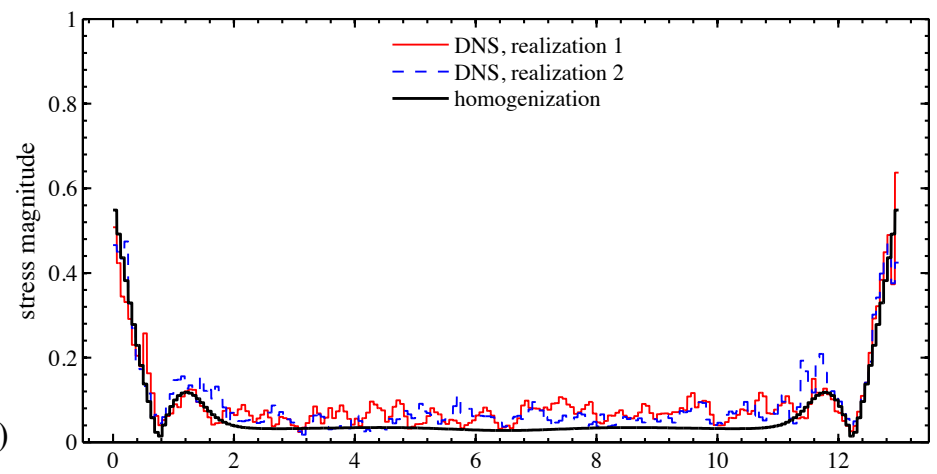

(b)

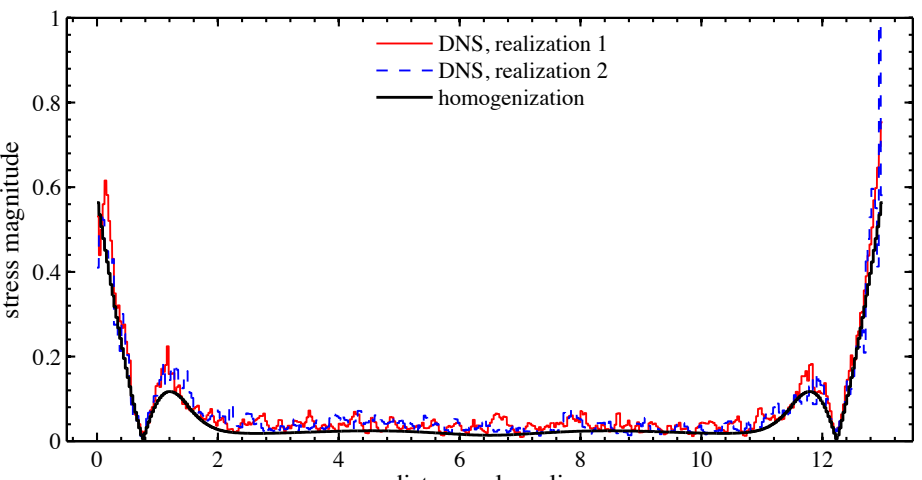

Figure 23: Stress magnitude, $\left(\sigma_{i j} \sigma_{i j}\right)^{1 / 2}$, along a line through the center of the I-beam (line 3 identified in Figure 17) for (a) a web thickness-to-grain ratio $=4$ using the $r 3$ mesh refinement, and (b) a web thickness-to-grain ratio $=8$ and using the $r 4$ mesh refinement. Results from two microstructural realizations are shown. Results obtained using the homogenized material properties are also shown. 
governed by the microstructural correlation length. Their analysis also discussed the presence of a boundary or surface effect in these nonlocal governing field equations. To investigate the presence of nonlocality in the ensemble-averaged stress field as well as surface effects, an ensemble consisting of 100 realizations of the microstructure within the I-beam is analyzed. Each of the 100 realizations of the model microstructure is obtained simply by changing the seed of the random number generator in both the MPS sampling described in Section 4.1 and in the assignment of grain orientations. The total number of grains in each realization varies by less than $0.1 \%$. For each finite element in the DNS mesh, the ensemble-average Cauchy stress tensor is calculated using an arithmetic average. Other tensorial averages are also possible [49].

The magnitude of the ensemble-averaged Cauchy stress along the three extraction lines/curves used in Section 5.4 are shown in Figures 24, 25, and 26, for both a web thickness-to-grain ratio of 4 and 8 . The homogenization results are also shown. The ensemble-averaged results are almost identical to the homogenization results, except for a small offset visible in Figure 25. Figure 25 shows the results along the edge of the hole at the end of the I-beam (curve 2 identified in Figure 17). There is also very little difference between the results for a web thickness-grain-ratio of 4 and a web thickness-grain-ratio of 8 . Thus, for the model polycrystalline microstructure any nonlocal effects or surface effects must be relatively small. Perhaps this result is not surprising since the correlation length for the model microstructure is less than the mean grain size as described in Section 4.1, although we expected to see some type of surface effect.

To investigate the statistical variation of the local stress field about the ensemble average, the sample maximum, minimum, and standard deviation are calculated at each point along the web-flange fillet. The point-wise sample maximum is somewhat sensitive to mesh refinement as described in Appendix A. To remove this sensitivity, each realization of the ensemble is first spatially filtered using the Gaussian moving average described in Section 5.6. The ensemble statistics of the stress magnitude of the filtered stress field at each point along the surface of the web-flange fillet are shown in Figure 27. Along the entire fillet, the stress variation about the ensemble mean is about $\pm 10 \%$ for the web thickness-to-grain ratio of 4 and $\pm 15 \%$ for the web thickness-to-grain ratio of 8 . Otherwise, the ensemble results for each web thickness-to-grain ratio are quite similar. 
(a)

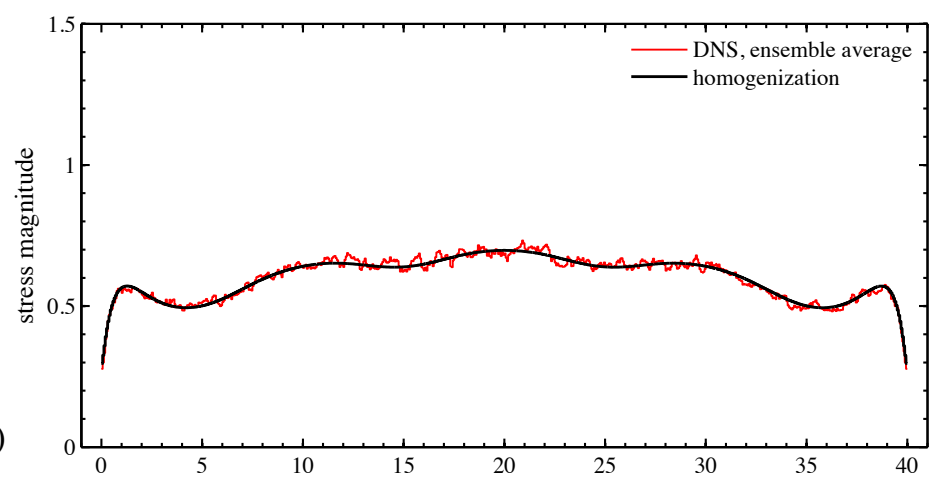

(b)

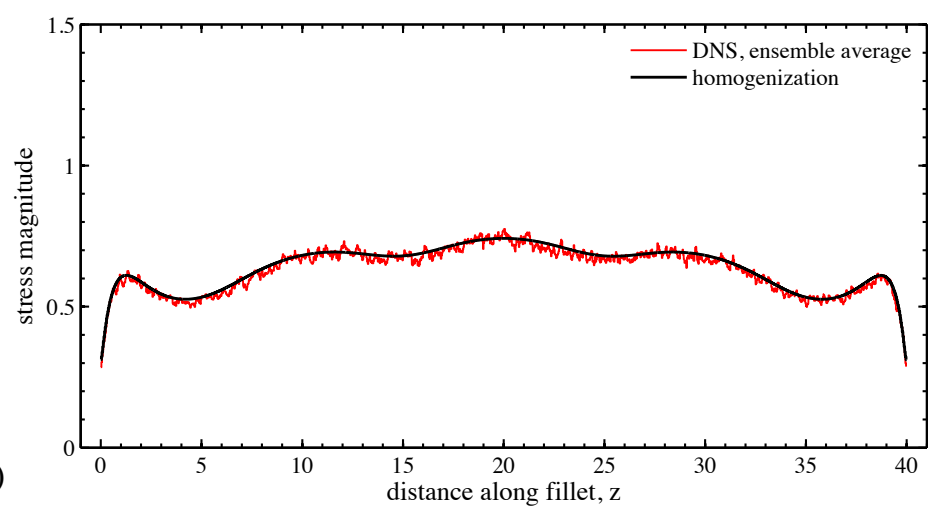

Figure 24: Magnitude of the ensemble-averaged stress tensor along the web-flange fillet of the I-beam (line 1 identified in Figure 17) for two thickness-to-grain ratios: (a) 4 and (b) 8. The ensemble consisted of 100 microstructural realizations. The stress magnitude obtained using the homogenized material properties is also shown. 
(a)

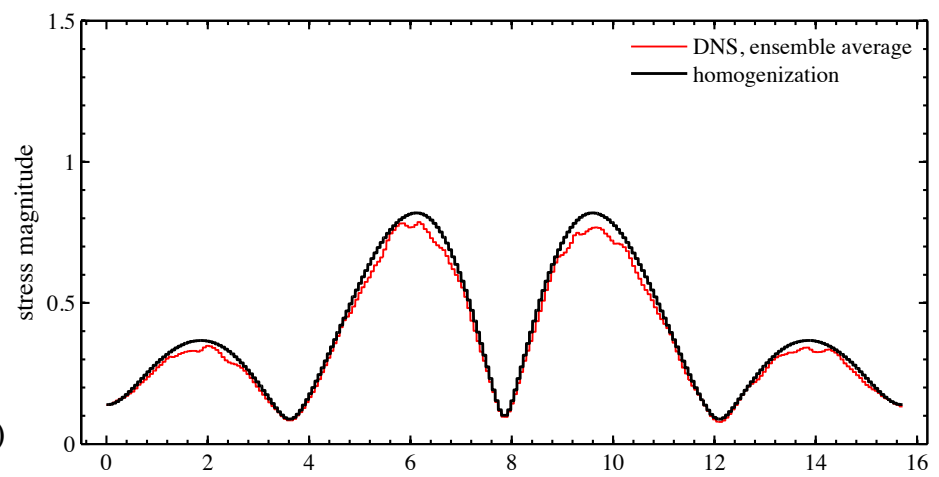

(b)

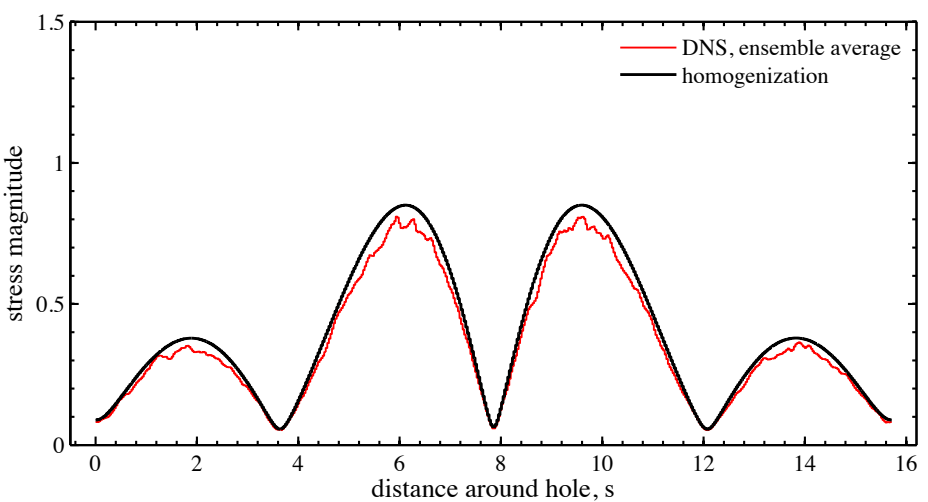

Figure 25: Magnitude of the ensemble-averaged stress tensor along the edge of the hole at the end of the I-beam (curve 2 identified in Figure 17) for the two thickness-to-grain ratios: (a) 4 and (b) 8. The ensemble consisted of 100 microstructural realizations. The stress magnitude obtained using the homogenized material properties is also shown. 
(a)

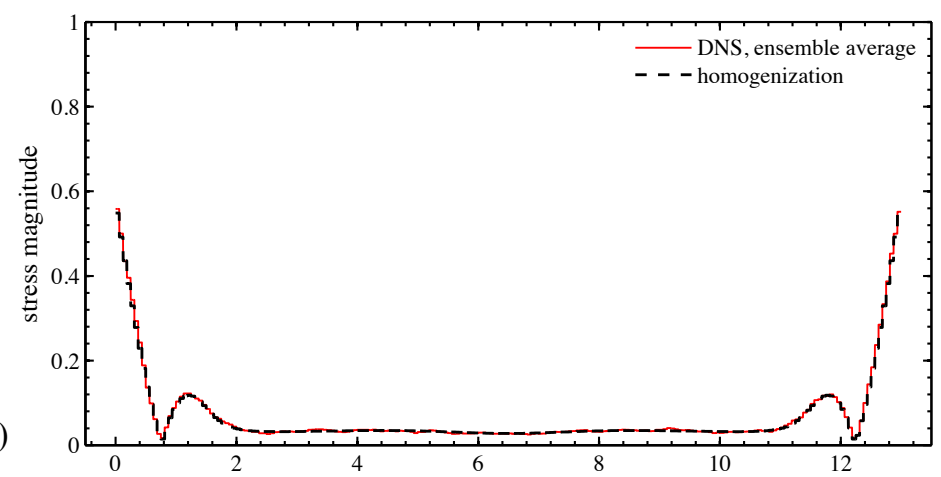

(b)

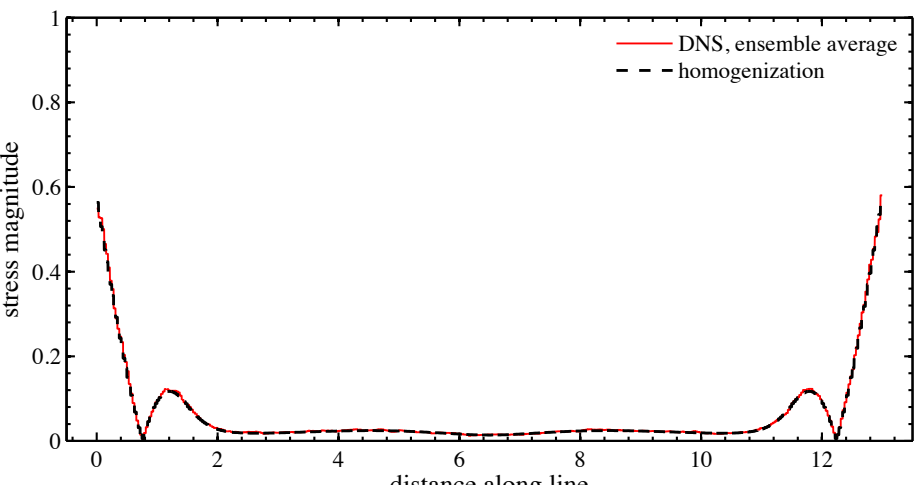

Figure 26: Magnitude of the ensemble-averaged stress tensor along a line through the center of the I-beam (line 3 identified in Figure 17) for the two thickness-to-grain ratios: (a) 4 and (b) 8. The ensemble consisted of 100 microstructural realizations. The stress magnitude obtained using the homogenized material properties is also shown. 
(a)

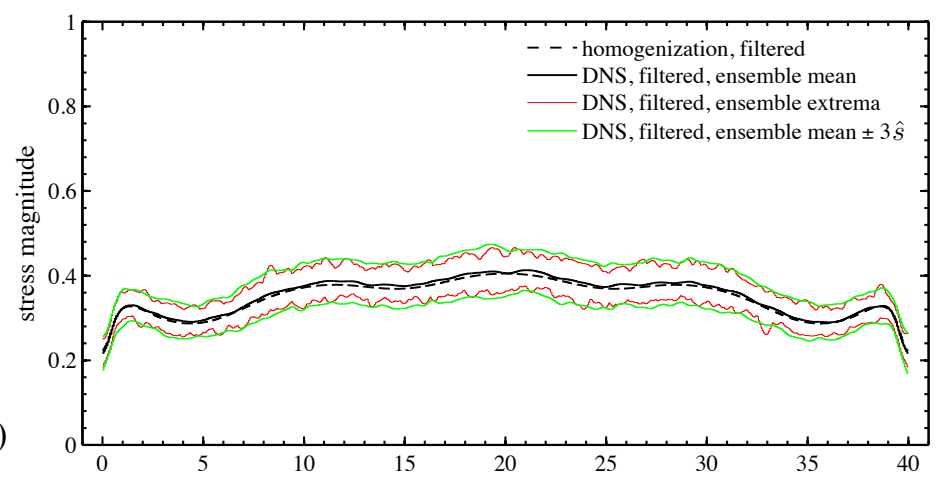

(b)

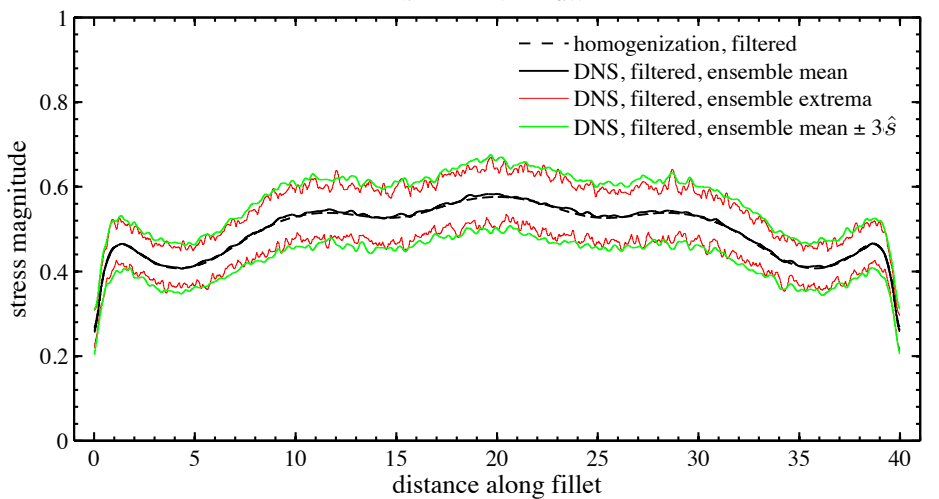

Figure 27: Ensemble statistics of the stress magnitude at each point along the surface of the web-flange fillet of the I-beam (line 1 identified in Figure 17) from the ensemble of 100 DNS simulations: (a) web thickness-to-grain ratio = 4 , and (b) web thickness-to-grain ratio $=8$. Ensemble mean, ensemble mean $\pm 3 \hat{s}$, and ensemble extrema are shown, where $\hat{s}$ is the sample standard deviation at a specific location. The stress field is first spatially filtered using the Gaussian moving average described in Section 5.6. 


\subsection{Spatial filter}

In this work, we are less interested in detailed stresses near the grain boundaries, but rather with the stress fluctuations above the grain scale. In order to investigate stress fluctuations above the grain scale, we use a spatial filter commonly used in imaging processing, namely the Gaussian filter [50]. The Gaussian filter is a weighted moving average and takes the form of a convolution in space,

$$
\hat{\sigma}_{i j}(\mathbf{x})=w_{\alpha}(\mathbf{x}) * \sigma_{i j}(\mathbf{x})=\int_{\mathbb{R}^{3}} w_{\alpha}(\mathbf{x}-\mathbf{y}) \sigma_{i j}(\mathbf{y}) d \mathbf{y},
$$

where $\hat{\sigma}_{i j}(\mathbf{x})$ is the filtered stress field, and $w_{\alpha}(\mathbf{x})$ is the Gaussian kernel given by (up to a normalization factor)

$$
w_{\alpha}(\mathbf{x}-\mathbf{y}) \propto e^{-\|\mathbf{x}-\mathbf{y}\|^{2} / \alpha^{2}} .
$$

Here, $\alpha$ is a spatial scaling parameter. The kernel function is normalized by requiring that the filter leaves a constant function unmodified. For a finite domain, this normalization factor will vary spatially near the boundary of the domain. This normalization factor is defined below for the discrete case. The Gaussian kernel is shown in Figure 28(a). The Gaussian filter has been used in the multiscale method of Yvonnet and Bonnet [51] to filter the microstructural-scale fields. The gain, $G(k)$, of a linear filter is the ratio of the signal output to the signal input, where $k$ is the magnitude of the spatial-frequency vector. It is given by the Fourier transform of $w_{\alpha}(\mathbf{x})$. For the Gaussian filter, $G(k)=e^{-\alpha^{2} k^{2} / 4}$. The cutoff frequency, $k_{c}$, is defined as the frequency at which the gain has a value of $1 / \sqrt{2}$. For the Gaussian filter, $k_{c}=\sqrt{2 \ln 2} / \alpha$. The gain of the Gaussian filter is shown in Figure 28(b).

In practice, the Gaussian kernel is truncated to have a finite support size. Beyond $x / \alpha \approx 2$, the kernel is approximately zero. To filter the DNS results presented in Section 5.4, the convolution in Eq. 12 is approximated using a discrete sum,

$$
\hat{\sigma}_{i j}(\mathbf{x}) \doteq \frac{1}{C(\mathbf{x})} \sum_{k \in I} w\left(\mathbf{x}-\mathbf{y}_{\mathbf{k}}\right) \sigma_{i j}\left(\mathbf{y}_{k}\right) V_{k},
$$

where $I$ represents the set of hexahedral elements whose centroids, $\mathbf{y}_{\mathbf{k}}$, are within a sphere of radius $2 \alpha$ about the point $\mathbf{x}, V_{K}$ is the volume of the hexahedral element, and $C(\mathbf{x})$ is the spatially varying normalization function. The normalization function $C(\mathbf{x})$ is given by

$$
C(\mathbf{x})=\sum_{k \in I} w\left(\mathbf{x}-\mathbf{y}_{\mathbf{k}}\right) V_{k} .
$$



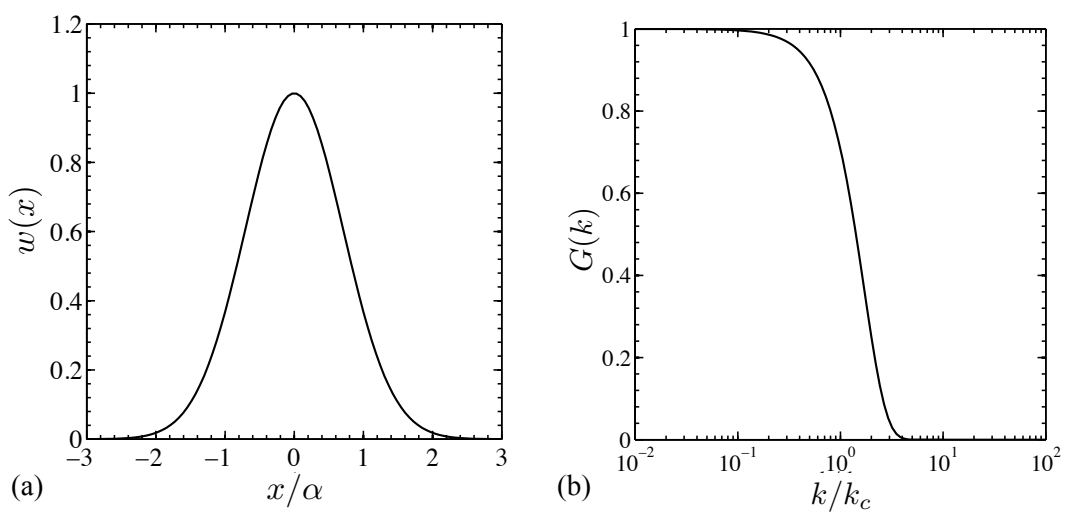

Figure 28: (a) Gaussian kernel, or weight function, $w(x)$, as a function of normalized and centered coordinate, $x / \alpha$, with $x=\|\mathbf{x}-\mathbf{y}\|$ (see Eq. 13). (b) Gain, $G(k)$, of the Gaussian filter as a function of normalized spatial-frequency vector, $k / k_{c}$, where $k$ is the magnitude of the spatial-frequency vector, and $k_{c}=\sqrt{2 \ln 2} / \alpha$ is the frequency at which the gain has the value of $1 / \sqrt{2}$.

Note that points in the interior of the domain away from the boundary will inherently have a larger discrete volume associated with the filter. Also, note that on infinite domains the exact Gaussian filter defined in Eq. 12 leaves both constant and linear functions unaltered. However, on finite domains the filter, both exact and discrete, is only capable of leaving constant functions unaltered. This boundary effect is a common issue with many filters [52]. Filters that leave linear functions unaltered on arbitrarily shaped domains can be developed using the Reproducing Kernel Method [53], which is beyond the scope of this paper.

The magnitude of the stress along the fillet of the I-beam, shown in Figure 19 for two microstructural realizations and two relative grain sizes, is shown again in Figure 29 after filtering the stress field. For the case of a web thickness-to-grain ratio of 4 , a value of $\alpha=0.25$ is used, which averages about 2 grain distances around each point. For the case of a web thickness-tograin ratio of 8 , a value of $\alpha=0.125$ is used to include about the same number of grains as used in the coarser microstructure. The homogenized solution is spatially filtered as well in order to have a meaningful comparison. There is very good agreement between the filtered DNS results and the homogenization results, except for the residual spatial fluctuations that have wavelengths of a few grain lengths.

The magnitude of the stress along the edge of the hole at the end of the I-beam, shown in Figure 21 for two microstructural realizations and two relative grain sizes, is shown again in 
(a)

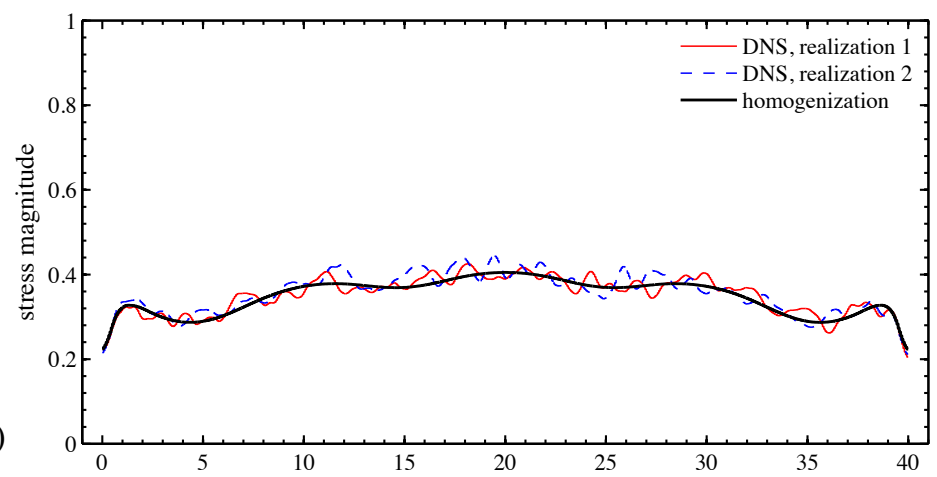

(b)

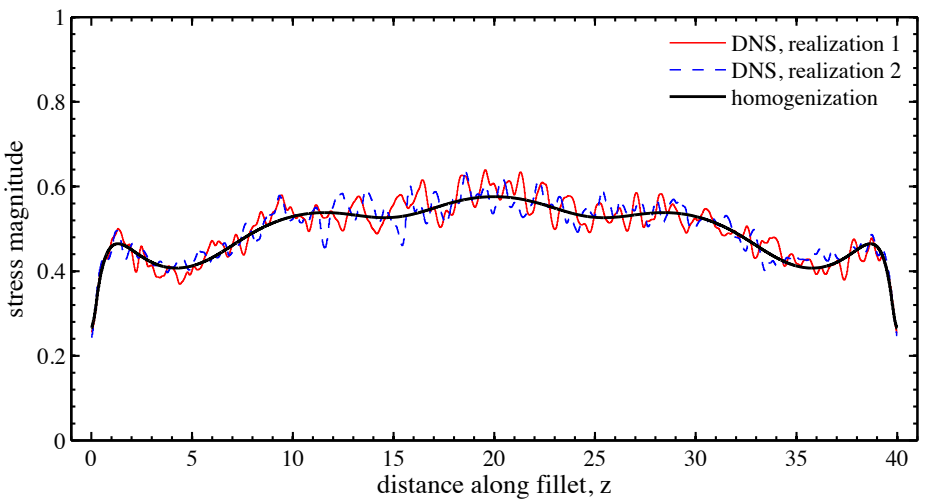

Figure 29: Magnitude of the filtered stress along the surface of the web-flange fillet of the I-beam (line 1 identified in Figure 17) for (a) a web thickness-to-grain ratio $=4$ using the $r 3$ mesh refinement with $\alpha=0.25$, and (b) a web thickness-to-grain ratio $=8$ using the $r 4$ mesh refinement with $\alpha=0.125$. Results from two microstructural realizations are shown. The filtered homogenization results are also shown. (cf. Figure 19.) 
(a)

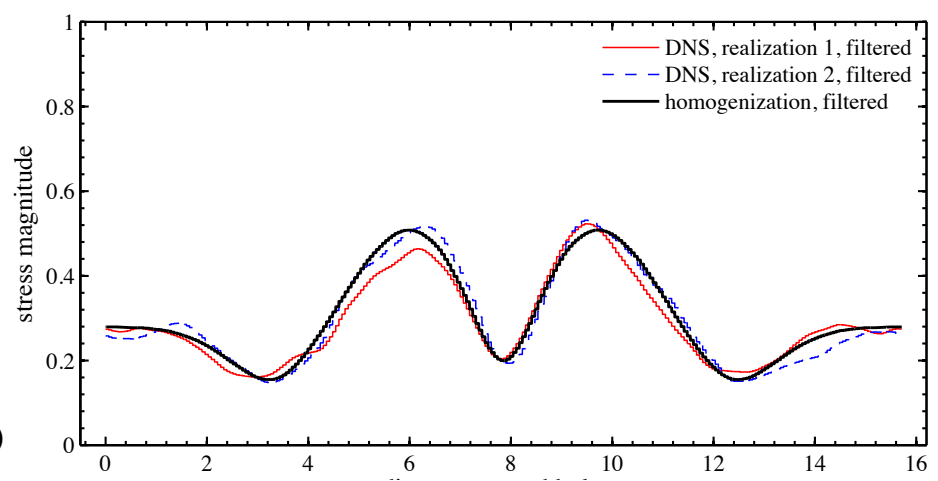

(b)

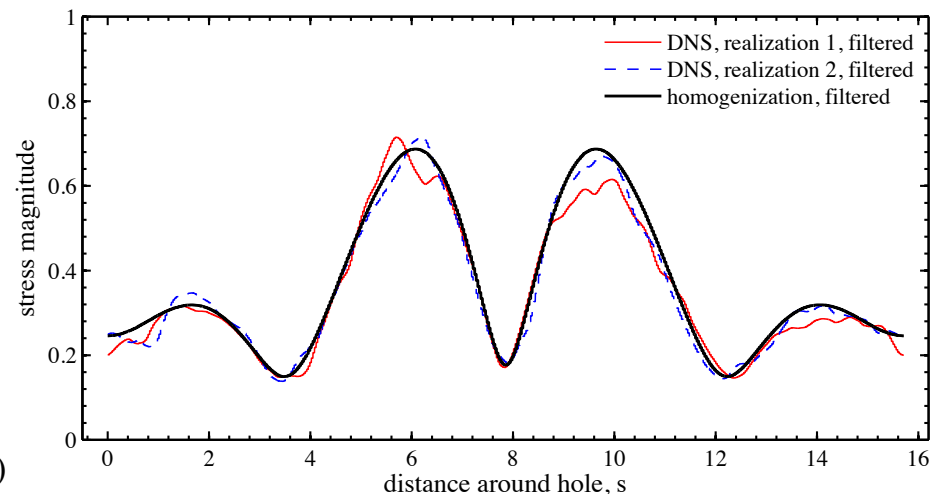

Figure 30: Magnitude of the filtered stress along the edge of the web-section hole at the end of the I-beam (curve 2 identified in Figure 17) for (a) a web thickness-to-grain ratio $=4$ using the $r 3$ mesh refinement with $\alpha=0.25$, and (b) a web thickness-to-grain ratio $=8$ and using the $r 4$ mesh refinement with $\alpha=0.125$. Results from two microstructural realizations are shown. The filtered homogenization results are also shown. (cf. Figure 21.)

Figures 30 after filtering the stress field. Here, the filtered results are quite smooth and almost equal to the homogenization results. While there are some local differences, there is reasonably good agreement between the filtered DNS results and the homogenization results for the two microstructural realizations considered here. Similar results are obtained for the extracted DNS stress distributions reported in Figure 23.

\section{Conclusions}

A fundamental challenge for the quantification of uncertainty in solid mechanics is understanding how microscale material variability is manifested at the macroscale. To aid in this understanding, we performed an ensemble of direct numerical simulations (DNS) in which polycrystalline microstructures were embedded throughout a macroscale structure. The macroscale 
structure was an I-beam with holes in the web section and fillets connecting the web and flanges. The microstructure was idealized using a Voronoi tessellation obtained from a maximal Poisson disk sampling process which resulted in an equiaxed grain structure. The orientation of each grain was taken to be an independent, identically distributed, random variable with no preferred orientation (no texture). This microstructure inherently has a correlation length that is less than the mean grain size. We investigated two relative grain sizes, namely 4 and 8 grains through the thickness of the web section. The DNS results were compared with corresponding simulations that used the homogenized material properties. Evidence was sought for surface effects and any higher-order effects due to the finite microstructure, both of which are predicted to exist by homogenization theory. The example material was an austenitic stainless steel, which possesses a relatively large elastic anisotropy ratio, making it a seemingly ideal material to display higher-order effects.

It was found that the displacement results using the homogenized material properties were an excellent approximation in a strong sense to the DNS results, despite the relatively large grain sizes used in the analysis. Additionally, the stress results using the homogenized material properties were an excellent approximation in a weak sense to both the ensemble-averaged stress field and the spatially filtered stress field of a single realization. Very little evidence was found for either higher-order effects or surface effects. This result is possibly due to the relatively small correlation length inherent in the model polycrystalline microstructure. One exception was around the end-hole in the web section. Here, a shift in the ensemble-averaged stress field compared to the homogenized solution was observed. Although not the focus of this study, the observed random fluctuations in the local stress field reinforce the importance of microstructural effects in predicting fatigue and fracture initiation.

\section{Appendix A. Effect of mesh refinement}

The DNS results presented in this paper use a constant mesh refinement level relative to the grain size. Namely, for a given mesh refinement that first resolved the grain size, two further mesh refinements are used. To investigate the effect of mesh refinement on the DNS results, the microstructural case of a web thickness-to-grain ratio of 4 is analyzed using the higher mesh refinement $r 4$ instead of $r 3$. Figure A.31 shows the microstructural realization originally shown in Figure 12 using the $r 3$ mesh refinement, but now using the $r 4$ refinement. Figure A.32 shows the 

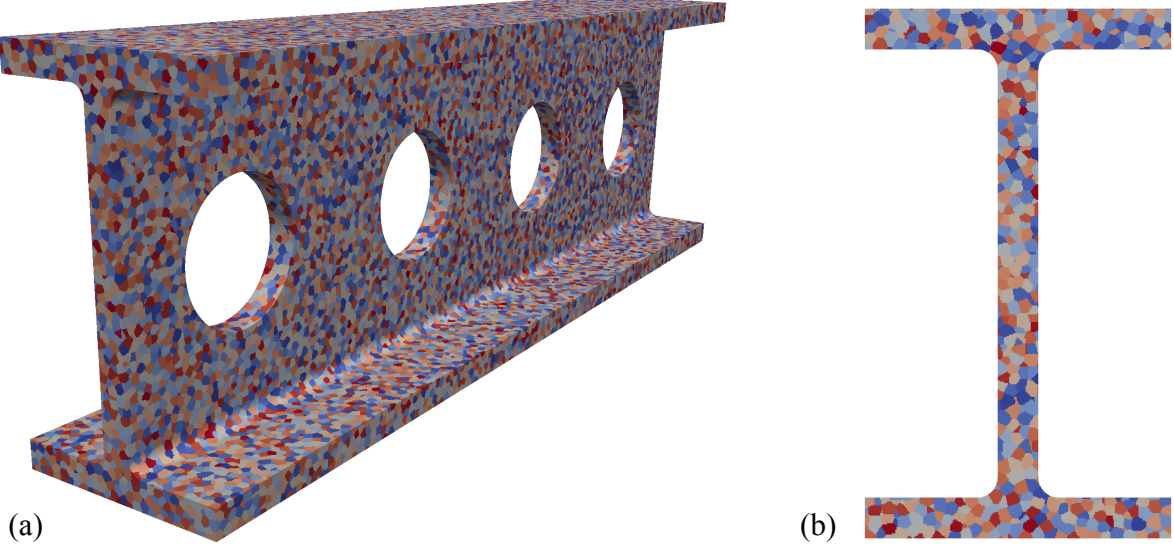

Figure A.31: (a) I-beam with embedded microstructure using the $r 4$ mesh refinement. The grain structure is identical to that shown in Figure 12, only the overlayed hexahedral mesh is finer ( $r 4$ instead of $r 3$ ). (b) A cross-section through the mid-section of the I-beam $(z=20)$.

resulting Von Mises stress field. This result should be compared with the Von Mises stress field shown in Figure 15. The extracted stress along the web-flange fillet is shown in Figure A.33(a) for both the $r 3$ and $r 4$ mesh refinements. The homogenization results using both mesh refinements are shown as well. The stress values do increase slightly, but the increase is similar to the increase in the homogenization results. Figure A.33(b) shows these same results but spatially filtered using the Gaussian moving average. For the spatially filtered results, there is very little difference between the $r 3$ and $r 4$ mesh refinements.

\section{Acknowledgments}

Sandia National Laboratories is a multi-program laboratory operated by Sandia Corporation, a wholly owned subsidiary of Lockheed Martin Corporation, for the U.S. Department of Energy's National Nuclear Security Administration under contract DE-AC04-94AL85000.

\section{References}

[1] U. Kocks, C. Tome, H. Wenk (Eds.), Texture and Anisotropy: Preferred Orientations in Polycrystals and their Effect on Material Properties, Cambridge University Press, New York, 1998.

[2] F. Roters, P. Eisenlohr, T. Bieler, D. Raabe, Crystal Plasticity Finite Element Methods: in Materials Science and Engineering, Wiley-VCH, Berlin, 2010. 


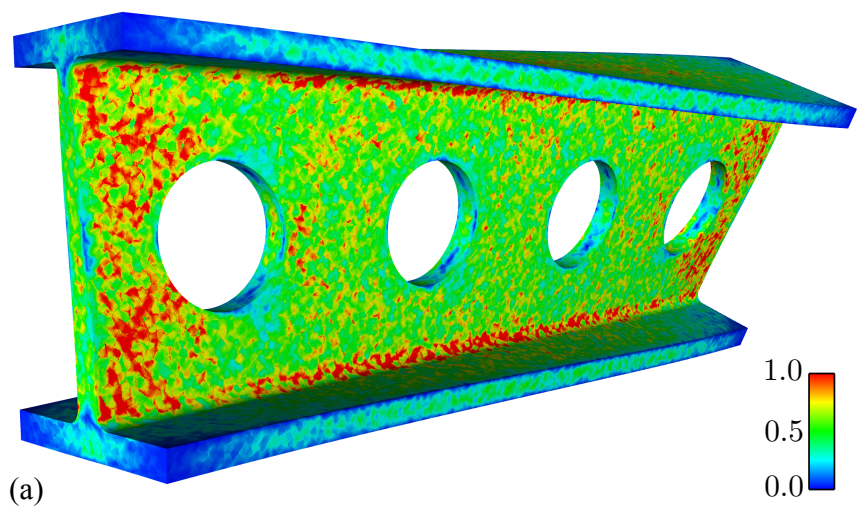

(b)

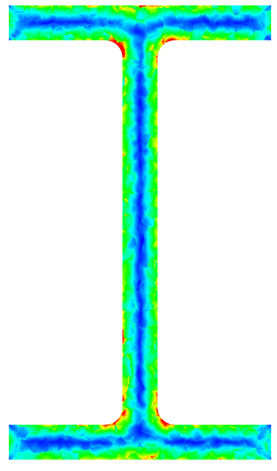

Figure A.32: (a) Von Mises stress field resulting from the direct numerical simulation of the microstructure shown in Figure A.31 using the $r 4$ mesh refinement. (b) Cross-sectional view of the Von Mises stress field at the mid-section of the I-beam $(z=20)$. (cf. Figure 15.)

(a)

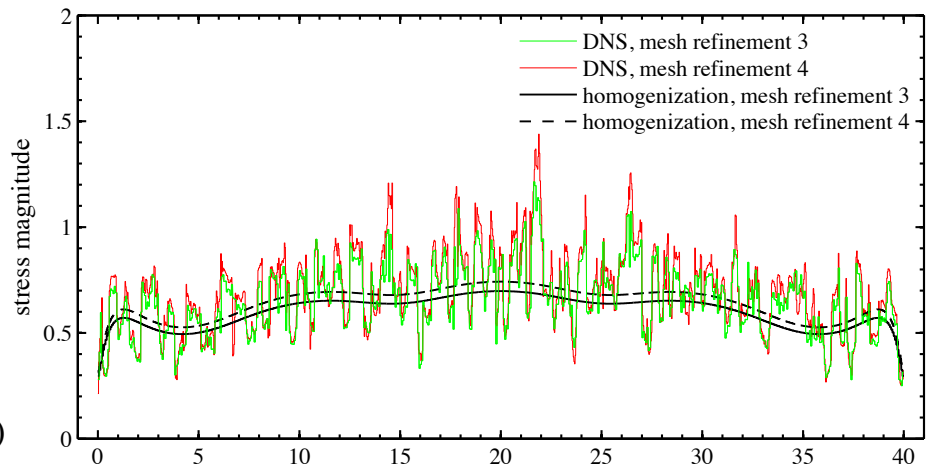

(b)

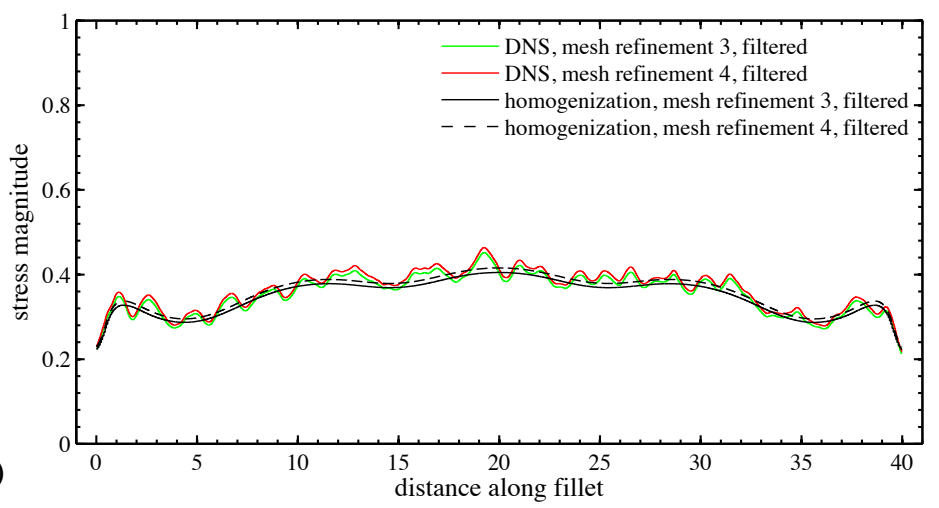

Figure A.33: (a) Stress magnitude along the surface of the web-flange fillet of the I-beam for a thickness-to-grain ratio = 4 and two mesh refinements, $r 3$ and $r 4$. Results obtained using the homogenized material properties are also shown. (b) The same results but spatially filtered. 
[3] J. Vetter (Ed.), Contemporary high performance computing: from petascale to exascale, Chapman and Hall/CRC, New York, 2013.

[4] S. Lee, G. Rohrer, A. Rollett, Three-dimensional digital approximations of grain boundary networks in polycrystals, Modelling and Simulation in Materials Science and Engineering 22 (2014) 025017.

[5] M. Veilleux, Geometrically explicit finite element modeling of AA7075-T651 microstructure with fatigue cracks, Ph.D. thesis, Cornell University, 2011.

[6] Y. Zhang, T. Hughes, C. Bajaj, An automatic 3D mesh generation method for domains with multiple materials, Computer Methods in Applied Mechanics and Engineering 199 (2010) 405-415.

[7] J. Qian, Y. Zhang, A. L. W. Wang, M. Qidwai, A. Geltmacher, Quality improvement of non-manifold hexahedral meshes for critical feature determination of microstructure materials, International Journal for Numerical Methods in Engineering 82 (2010) 1406-1423.

[8] C. Wang, Direct extraction of surface meshes from implicitly represented heterogeneous volumes, Modelling and Simulation in Materials Science and Engineering 39 (2007) 35-50.

[9] D. Cioranescu, P. Donato, An Introduction to Homogenization, Oxford University Press, Oxford, 1999.

[10] R. Mindlin, Micro-structure in linear elasticity, Archive for Rational Mechanics and Analysis 16 (1964) 51-78.

[11] V. Smyshlyaev, K. Cherednichenko, On rigorous derivation of strain gradient effects in the overall behaviour of periodic heterogeneous media, Journal of the Mechanics and Physics of Solids 48 (2000) 1325-1357.

[12] T. Tran, V. Monchiet, G. Bonnet, A micromechanics-based approach for the derivation of constitutive elastic coefficients of strain-gradient media, International Journal of Solids and Structures 49 (2012) 783-792.

[13] X. Yuan, Y. Tomita, T. Andou, A micromechanical approach of nonlocal modeling for media with periodic microstructures, Mechanics Research Communications 35 (2008) 126-133.

[14] J. Auriault, G. Bonnet, Surface effects in composite materials: Two simple examples, International Journal of Engineering Science 25 (1987) 307-323.

[15] H. Dumontet, Study of a boundary layer problem in elastic composite materials, Mathematical Modelling and Numerical Analysis 20 (1987) 265-286.

[16] F. Devries, H. Dumontet, G. Duvaut, F. Lene, Homogenization and damage for composite structures, International Journal for Numerical Methods in Engineering 27 (1989) 285-298.

[17] M. Beran, J. McCoy, Mean field variations in a statistical sample of heterogeneous linearly elastic solids, International Journal of Solids and Structures 6 (1970) 1035-1054.

[18] W. Drugan, J. Willis, A micromechanics-based nonlocal constitutive equation and estimates of representative volume element size for elastic composites, Journal of the Mechanics and Physics of Solids 44 (1996) 497-524.

[19] R. Zallen, The Physics of Amorphous Solids, Wiley-VCH, Berlin, 2004. Ch. 2.

[20] I. Temizer, P. Wriggers, An adaptive multiscale resolution strategy for the finite deformation analysis of microheterogeneous structures, Computer Methods in Applied Mechanics and Engineering 200 (2011) 2639-2661.

[21] E. Sanchez-Palencia, Non-Homogeneous Media and Vibration Theory, Lecture Notes in Physics, vol. 127, Springer-Verlag, 1980.

[22] V. Jikov, S. Kozlov, O. Oleinik, Homogenization of differential operators and integral functionals, Springer-Verlag, New York, 1994

[23] A. Bensoussan, J. Lions, G. Papanicolaou, Asymptotic analysis for periodic structures, American Mathematical 
Society, 2011

[24] G. Papanicolaou, S. Varadhan, Boundary value problems with rapidly oscillating random coefficients, Colloquia Mathematica Societatis J'anos Bolyai 27 (1979) 835-873.

[25] C. Boutin, Microstructural effects in elastic composites, International Journal of Solids and Structures 33 (1996) 1023-1051.

[26] T. Kanit, S. Forest, I. Galliet, V. Mounoury, D. Jeulin, Determination of the size of the representative volume element for random composites: statistical and numerical approach, International Journal of Solids and Structures 40 (2003) 3647-3679.

[27] P. Eisenlohr, M. Diehl, R. Lebenshohn, F. Roters, A spectral method solution to crystal elasto-viscoplasticity at finite strains, International Journal of Plasticity 46 (2013) 37-53.

[28] D. Pyle, J. Lu, D. Littlewood, A. Maniatty, Effect of 3D grain structure representation in polycrystal simulations, Computational Mechanics 52 (2013) 135-150.

[29] Sierra/SolidMechanics 4.22 Users Guide, Technical Report SAND2011-7597, Sandia National Laboratories, 2011

[30] C. Farhat, M. Lesoinne, P. LeTallec, K. Pierson, D. Rixen, FETI-DP: a dual-primal unified FETI method part I: A faster alternative to the two-level FETI method, International Journal for Numerical Methods in Engineering 50 (2001) 1523-1544.

[31] CUBIT Geometry and Meshing Toolkit, Version 13.2, https://cubit.sandia.gov/public/13.2/ Cubit-13.2-announcement.html, 2012.

[32] H. Ledbetter, Monocrystal-polycrystal elastic constants of a stainless steel, Physica Status Solidi (a) 85 (1984) 89-96.

[33] A. Bower, Applied Mechanics of Solids, CRC Press, New York, 2010. Ch. 3.2.16.

[34] J. Madison, H. Jin, J. Foulk, S. Murawski, C. Robino, New microstructural insights to the constitutive behavior of laser welds in 304L stainless steel - I, Metallurgical and Materials Transactions A (2014). (in preparation).

[35] M. Ebeida, S. Mitchell, A. Patney, A. Davidson, J. Owens, A simple algorithm for maximal poisson-disk sampling in high dimensions, Computer Graphics Forum 31 (2012) 785-794.

[36] J. Finney, Random packings and the structure of simple liquids. 1. the geometry of random close packing, Proceedings of the Royal Society of London A 319 (1970) 479-493.

[37] J. Arvo, Fast random rotation matrices, in: D. Kirk (Ed.), Graphics Gems III, Academic Press, New York, 1992. Ch. 3.4.

[38] X. Yin, W. Chen, A. To, C. McVeigh, W. Liu, Statistical volume element method for predicting microstructureconstitutive property relations, Computer Methods in Applied Mechanics and Engineering 197 (2008) 3516-3529.

[39] A. Gusev, Representative volume element size for elastic composites: A numerical study, Journal of the Mechanics and Physics of Solids 45 (1997) 1449-1459.

[40] M. Ostoja-Starzewski, Random field models of heterogeneous materials, International Journal of Solids and Structures 35 (1998) 2429-2455.

[41] Z. Ren, Q. Zheng, A quantitative study on minimum size of representative volume elements of cubic polycrystals - numerical experiments, Journal of the Mechanics and Physics of Solids 50 (2002) 881-893.

[42] Z. Ren, Q. Zheng, Effects of grain sizes, shapes, and distribution on minimum sizes of representative volume elements of cubic polycrystals, Mechanics of Materials 36 (2004) 1217-1229. 
[43] C. Huet, Application of variational concepts to size effects in elastic heterogeneous bodies, Journal of the Mechanics and Physics of Solids 38 (1990) 813-841.

[44] E. Kreysig, Introductory functional analysis with applications, John Wiley and Sons, New York, 1978. Ch. 4.1.

[45] S. Resnick, A Probability Path, Birkhauser, Boston, 1999. Ch. 8.6.1.

[46] M. Moakher, A. Norris, The closest elastic tensor of arbitrary symmetry to an elasticity tensor of lower symmetry, Journal of Elasticity 85 (2006) 215-263.

[47] L. Richardson, J. Gaunt, The deferred approach to the limit, Philosophical Transactions of the Royal Society A 226 (1927) 299-349.

[48] H. Ledbetter, N. Frederick, M. Austin, Elastic constant variability in stainless steel 304L, Journal of Applied Physics 51 (1980) 305-309.

[49] M. Moakher, On the averaging of symmetric positive-definite tensors, Journal of Elasticity 82 (2006) 273-296.

[50] M. Nixon, A. Aguado, Feature Extraction and Image Processing for Computer Vision, Academic Press, New York, second edition, 2012. Ch. 3.4.4.

[51] J. Yvonnet, G. Bonnet, A consistent nonlocal scheme based on filters for the homogenization of heterogeneous linear materials with non-seperate scales, International Journal of Solids and Structures 51 (2014) 196-209.

[52] Y. Ge, Q. Cheng, Boundary effect reduction in image filtering, International Journal on Graphics, Vision, and Image Processing 7 (2007) 17-25.

[53] Y. You, J. Chen, H. Lu, Filters, reproducing kernel, and adaptive meshfree method, Computational Mechanics 31 (2003) 316-326. 